\title{
Analysis of Fluid Flow and Wall Shear Stress Patterns Inside Partially Filled Agitated Culture Well Plates
}

\author{
M. Mehdi Salek, ${ }^{1,2}$ Pooria Sattari, ${ }^{2}$ and Robert J. Martinuzzi ${ }^{1,2}$ \\ ${ }^{1}$ Biofilm Engineering Research Group, Calgary Center for Innovative Technology, University of Calgary, Calgary, AB, Canada; \\ and ${ }^{2}$ Department of Mechanical and Manufacturing Engineering, University of Calgary, 2500 University Drive N.W., \\ Calgary, AB T2N 1N4, Canada
}

(Received 8 September 2011; accepted 10 October 2011)

Associate Editor Konstantinos Konstantopoulo oversaw the review of this article.

\begin{abstract}
The appearance of highly resistant bacterial biofilms in both community and hospitals environments is a major challenge in modern clinical medicine. The biofilm structural morphology, believed to be an important factor affecting the behavioral properties of these "super bugs", is strongly influenced by the local hydrodynamics over the microcolonies. Despite the common use of agitated well plates in the biology community, they have been used rather blindly without knowing the flow characteristics and influence of the rotational speed and fluid volume in these containers. The main purpose of this study is to characterize the flow in these high-throughput devices to link local hydrodynamics to observed behavior in cell cultures. In this work, the flow and wall shear stress distribution in six-well culture plates under planar orbital translation is simulated using Computational Fluid Dynamics (CFD). Free surface, flow pattern and wall shear stress for two shaker speeds (100 and $200 \mathrm{rpm}$ ) and two volumes of fluid (2 and $4 \mathrm{~mL}$ ) were investigated. Measurements with a non-intrusive optical shear stress sensor and High Frame-rate Particle Imaging Velocimetry (HFPIV) are used to validate CFD predictions. An analytical model to predict the free surface shape is proposed. Results show a complex three-dimensional flow pattern, varying in both time and space. The distribution of wall shear stress in these culture plates has been related to the topology of flow. This understanding helps explain observed endothelial cell orientation and bacterial biofilm distributions observed in culture dishes. The results suggest that the mean surface stress field is insufficient to capture the underlying dynamics mitigating biological processes.
\end{abstract}

Keywords-Six-well culture plates, CFD, Hydrodynamics, Wall shear stress, Optical shear stress sensor, PIV, Biofilms, Endothelial cells.

Address correspondence to Robert J. Martinuzzi, Department of Mechanical and Manufacturing Engineering, University of Calgary, 2500 University Drive N.W., Calgary, AB T2N 1N4, Canada. Electronic mail: rmartinu@ucalgary.ca

\section{INTRODUCTION}

The appearance of biofilms, communities of bacterial cells developed on either an inert or living surface and encased in a self-generated exopolysaccharide (EPS) matrix, is one of the major challenges in modern clinical medicine. Because of the natural resistance of these complex structures against different types of antimicrobial agents, they are associated with chronic wound infections, a variety of recalcitrant diseases (e.g., cystic fibrosis) and infections of many medical devices (e.g., heart valves, implants, and urinary catheters) often resulting in device replacement and increased treatment costs. ${ }^{4,5,8,18-23}$ Hence, a large number of studies have been devoted to finding new solutions to eradicate these super bugs and prevent their formation.

Several studies have confirmed the importance of the surrounding hydrodynamics on the formation process and ensuing biofilm morphology. ${ }^{6,24,25,32}$ In turn, the morphology has been associated with different phenotypic expression. ${ }^{12,25,29,30}$ Hence, an accurate knowledge of the local hydrodynamic conditions is important for correctly interpreting biological observations.

The prediction of the flow patterns and parameters such as wall shear stress in different types of ducts and parallel plate flow cells is not a difficult task; however, the use of these flow cells is not practical when a large number of concurrent tests or identical replicates are needed. Furthermore, a major clinical challenge is to identify both the bacterial strain and an effective antibiotic combination under the correct flow conditions, which may require several hundred independent tests within a short time (i.e., before the infection develops beyond control, usually $24 \mathrm{~h}$ ). Various types of standard high-throughput devices 
are commonly used for this purpose allowing rapid production of biofilms and running multiple tests in parallel. These devices usually consist of a plate with a number of identical wells. When the plate is translated by an orbital shaker, it provides the same hydrodynamic condition in all of the wells. An additional advantage of these devices is that the hydrodynamic conditions can be altered by changing the shaker motion.

Six-well plates are one type of the high-throughput devices widely used in the microbiology community due to their practicality. ${ }^{2,15,28,31}$ These compact devices, facilitate multi-sample testing, provide oscillatory fluid motion with adjustable speeds and do not require bulky pump-flow cell-tubing setups and problems associated with them in terms of manufacturing and sealing. Also, the relatively large and flat surfaces of each well provide easy access for optical measurements, microscopy and other analysis. Therefore, utilizing these dishes for hydrodynamic studies, including parameters such as shear stress and mass transport, is potentially of great advantage. However, unless the fluid mechanics in these containers is well resolved, interpreting and comparing the effect of parameters with confidence is difficult.

Among the few attempts to clarify the hydrodynamic characteristics in six-well plates, Azevedo et al. ${ }^{2}$ modeled the flow inside these wells to study the effects of shear stress on the adhesion of Helicobacter pylori to stainless steel. This study was limited to the estimation of an overall mean wall shear stresses at the bottom of the well. As will be discussed later, the periodic flow inside these wells is complex due to the presence of side walls. Noticeable differences exist between regions of the well both in terms of the local mean and fluctuating wall shear stress amplitude. It has been shown in an earlier study ${ }^{15}$ that biofilm deposition and morphology in these plates is non-uniform and the biofilm characteristics correlate strongly with local shear stress mean and fluctuation levels.

The flow in a six-well container undergoing orbital motion in a plane normal to the gravitational vector is complicated by the presence of the container side walls and the shallow liquid fill. For an unbounded fluid, the fluid motion induced by the orbiting plate is a simple variation of Stokes' Second Problem, which is governed by a linear differential equation. Hence, the fluid motion induced by plate undergoing orbital translation is obtained simply by a superposition of the solution in the two orthogonal directions (Sherman ${ }^{27}$ ). Briefly, letting $x, z$ represent the co-ordinates in the horizontal plane, $\vec{i}, \vec{k}$, the corresponding unit vectors and $y$ the co-ordinate in the vertical direction, for a solid plate executing the motion $u(0, t)=-U_{0}(\cos (\Omega t) \vec{i}+$ $\sin (\Omega t) \vec{k})$ the velocity distribution is given by:

$$
u(y, t)=-U_{0}[\cos (\Omega t-\lambda y) \vec{i}+\sin (\Omega t-\lambda y) \vec{k}]
$$

such that at any point on the plate, the magnitude of the velocity and shear stress are independent of time:

$$
\|U(y)\|=U_{0} e^{-\lambda y} \quad \tau_{w}=-\mu U_{0}\left(\frac{\Omega}{v}\right)^{1 / 2}
$$

where $\lambda=\sqrt{\Omega / 2 v}, \Omega$ is the angular orbital speed; $U_{0}$ is the maximum plate speed; $\mu, v$ are the kinematic and dynamic viscosities. Note that the viscous effects are concentrated in the Ekman layer, $\delta \cong \frac{4.61}{\lambda}$ (defined for $\left.\|U(\delta)\|=0.01 U_{0}\right)$, above which the fluid behaves as inviscid.

The movement of the vertical side walls of a confined fluid in a container induces a rotation of the fluid. While the problem of a fluid in a container undergoing constant solid body rotation has been extensively studied for laminar ${ }^{10,11}$ and turbulent ${ }^{13,14}$ regimes, the present problem is more related to the spin-up problem, since the solid boundaries are rotating relative to the fluid. The impulsively started transient problem in laminar regime has been analytically resolved ${ }^{10,11,13}$ for deep fluids (fluid heights much greater than the Ekman layer). It has been shown that the viscous effects are mainly concentrated in the near wall layers, such that most of the fluid can be treated as inviscid with a columnar velocity distribution. In the case of the present geometry, however, the fluid layer is shallow and the shape of the free surface plays a preponderant role in determining the flow patterns such that earlier treatments are not directly amenable.

Six-well plates are also commonly used in endothelial cell studies. Dardik et al. ${ }^{7}$ used these plates to study the effects of orbital shear stress on endothelial cells. Experimental data over a range of shaker rotation speeds was reported, but due to low data rates, the time variation in the shear stress could not be resolved. Berson et $\mathrm{al}^{3}$ performed a numerical study of flow within these culture dishes to investigate the influence of wall shear stress on endothelial cells. This work did show that mean shear stress levels at the bottom of the plate were not uniform and did not correspond well to a simple analytical "oscillating plate" model and that the rotational speed influenced the radial shear stress distribution. Although this work and that of Kostenko et al. ${ }^{15}$ provided some insight into the wall shear stress distribution on the bottom faces of six-well plates, they also highlight an understanding of flow physics leading to the observed shear stress patterns is lacking. For example, morphological differences observed in regions with similar mean shear stress levels were found to correlate better with shear fluctuation levels, for which the distribution appeared related to the orbital speed and liquid fill volume affecting the flow 
pattern. A systematic investigation to characterize how the basic operating parameters (i.e., shaker speed and liquid volume) influence the flow patterns and wall shear stress inside the wells could thus be valuable for controlling or predicting the hydrodynamic conditions in an orbiting well. This capability can allow for better experimental design and more reliable interpretation of biological test results.

The instantaneous flow pattern and wall shear stress distribution inside a well translated by an orbital shaker was investigated for different shaker speeds and volumes of fluid typically used in microbiology studies. The numerical simulations of the flow field were validated by experimental measurements. The mechanisms giving rise to the shear stress distribution and shape of the free surface are discussed in detail. These findings are then related to the orientation of endothelial cells and distribution of bacterial biofilms cultured in sixwell plates.

\section{METHODS}

\section{Numerical Model and Governing Equations}

The unsteady, free-surface flow inside a well of a sixwell culture plate was simulated using the finite volume based, commercial Computational Fluid Dynamics (CFD) software FLUENT ver. 6.3. ${ }^{9}$ Two rotational speeds of the orbital shaker, i.e., 100 and $200 \mathrm{rpm}$, and two liquid volumes, i.e., 2 and $4 \mathrm{~mL}$ (four cases in total) were simulated. The free surface motion in the liquid-air interface, the flow patterns inside the well and the mean and instantaneous wall shear stresses were analyzed.

The six-well culture plate consists of six identical wells shown schematically in Fig. 1a. Each well is $18 \mathrm{~mm}$ deep and has a radius of $R=17.5 \mathrm{~mm}$. The wells are mounted on an orbital shaker and undergo a two-dimensional linear translation in the horizontal plane (i.e., $X Z$ plane). The radius of gyration is
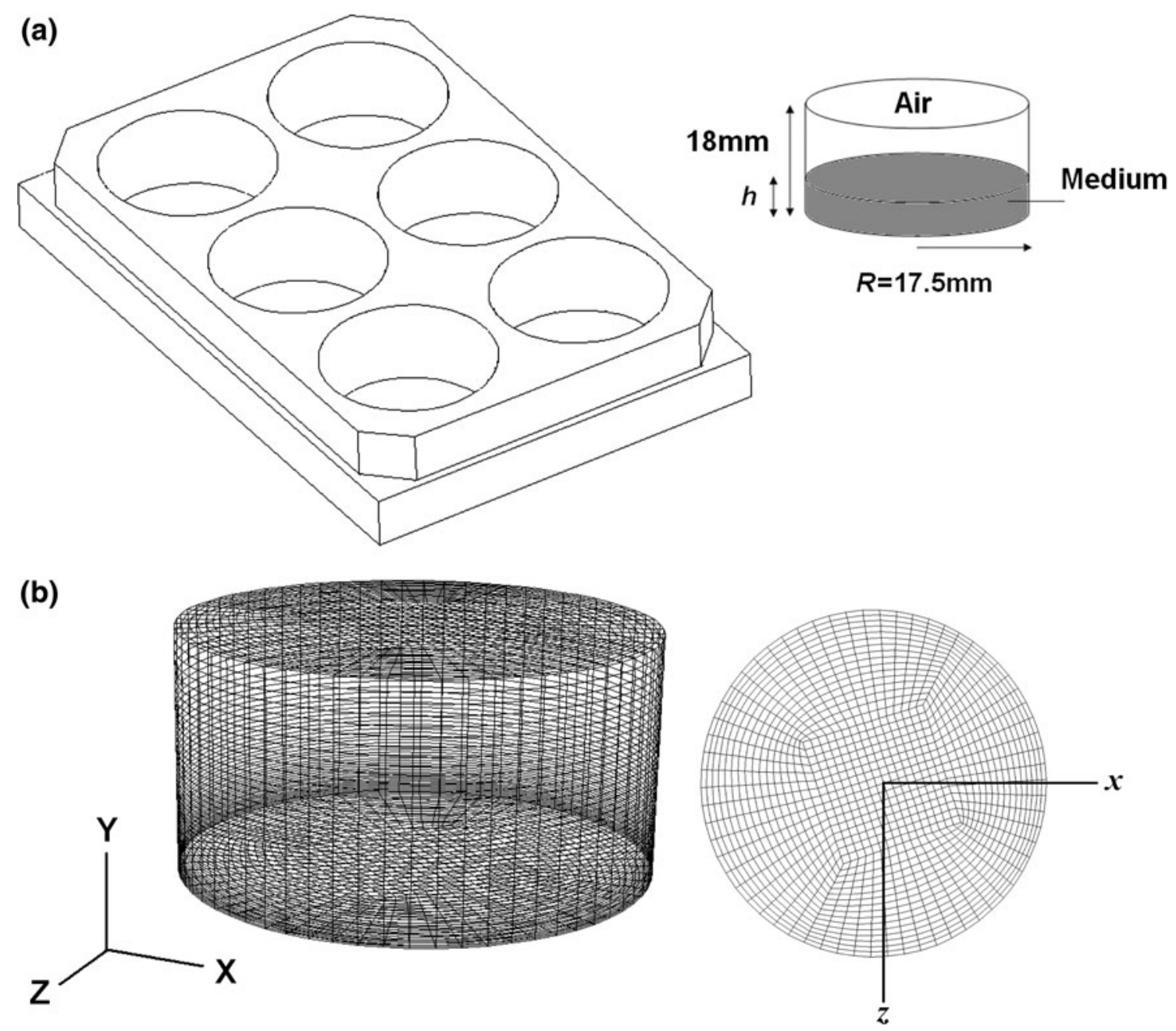

FIGURE 1. (a) Isometric diagram of six-well culture plate and schematic of a single well geometry; (b) view of computational grid in a single well of a six-well plate. $X, Y, Z$ denote the stationary reference frame and $x, y, z$ denote the moving reference frame. 
$R_{\mathrm{g}}=9.5 \mathrm{~mm}$. The properties of water are used to model the liquid phase and air to model the gas phase. All properties are at $20^{\circ} \mathrm{C}$ and $1 \mathrm{~atm}: \rho=998.2 \mathrm{~kg} /$ $\mathrm{m}^{3}, \mu=0.001003 \mathrm{~Pa} \mathrm{~s}$. Each well is filled with 2 or $4 \mathrm{~mL}$ of liquid medium, corresponding to approximately 2 and $4 \mathrm{~mm}$ liquid height when at rest. The Reynolds number is defined as $R e=\rho \Omega R_{\mathrm{g}}^{2} / \mu$ following Dardik et l. $^{7}$ The maximum Re in this study is 1886 such that the flow is expected to remain in the laminar regime.

To simulate this two-phase flow, the solution domain is divided into three regions: two regions corresponding to the homogeneous single-phase fluids (air and water) and a two-phase water-air interface. The three-dimensional unsteady mass and momentum conservation equations in the homogeneous domain are written, respectively, as:

$$
\begin{gathered}
\frac{\partial \rho}{\partial t}+\nabla \cdot(\rho \vec{v})=0 \\
\frac{\partial}{\partial t}(\rho \vec{v})+\nabla \cdot(\rho \vec{v} \vec{v})=-\nabla p+\nabla(\mu \nabla \vec{v})+\rho \vec{F}
\end{gathered}
$$

where $\vec{v}, \rho, \mu, p$, and $\vec{F}$ are velocity, density, dynamic viscosity, pressure, and external force (per unit mass) for the corresponding single-phase, respectively.

To capture the free surface flow, the volume of fluid (VOF) method $^{9}$ was used, such that the three domains could be combined into a continuous domain for the solution procedure. This approach insures that the dynamic condition (shear stress at the free-surface is equal for both phases) is satisfied. It is assumed that each fluid phase domain is simply connected. In each computational finite volume at the interface, the volume fraction for each phase, $\alpha_{i}$, is introduced satisfying:

$$
\sum_{i=1}^{n} \alpha_{i}=1
$$

where $i$ represents air or water (i.e., $n=2$ ). The continuity and momentum equations are then solved using a modified definition for the fluid properties:

$$
\psi=\sum_{\substack{i=\text { air, } \\ \text { water }}} \alpha_{i} \psi_{i}
$$

where $\psi$ can be the density or the dynamic viscosity. The system of equations is then closed ${ }^{9}$ by satisfying:

$$
\frac{\partial \alpha_{i}}{\partial t}+\vec{v} \cdot \nabla \alpha_{i}=0
$$

The geometric Reconstruct Scheme, which is the most accurate interface tracking technique, ${ }^{9}$ was employed for the calculation of the transient VOF model. It uses a Piecewise-linear approach assuming that the interface between the two phases has a linear slope in each cell. ${ }^{35}$
In the aforementioned treatment, it is assumed that the influence of surface tension $(\sigma=0.072 \mathrm{~N} / \mathrm{m}$ for water-air interface at $20^{\circ} \mathrm{C}$ ) is negligible. This assumption is valid if the gravitational forces and inertial forces on the liquid phase, expressed through the Bond $\left(B o=\frac{\left(\rho_{\text {water }}-\rho_{\text {air }} g(2 R)^{2}\right.}{\sigma}\right)$ and Webber $\left(W e=\frac{\rho_{\text {water }} U^{2}(2 R)}{\sigma}\right)$ numbers, respectively, are significantly larger than the capillary forces (i.e., $B o$, $W e \gg 1)$. In the present study, $B o$ and $W e$ are typically of the order of 100 .

\section{MOTION OF THE SIX-WELL PLATE AND BOUNDARY CONDITIONS}

The orbital shaker imparts the same two-dimensional, in-plane movement to all points on the six-well plate. The velocity of the plate walls are thus given by:

$$
\vec{U}=\left[\begin{array}{c}
U_{x} \\
U_{y} \\
U_{z}
\end{array}\right]=\left[\begin{array}{c}
-R_{\mathrm{g}} \Omega \sin \Omega t \\
0 \\
-R_{\mathrm{g}} \Omega \cos \Omega t
\end{array}\right]
$$

where $R_{\mathrm{g}}=9.5 \mathrm{~mm}$ is the orbital radius of agitation and $\Omega$ is its rotational speed.

The solution domain encloses both fluid phases and extends to the upper lip of the well, although only the results in the liquid layer are presented. A no-slip boundary condition was imposed at the solid walls of the six-well container (i.e., no relative velocity between the fluid and the solid surfaces).

The equations of motion were solved in both a stationary and in a moving frame of reference for comparison and validation. For the stationary reference frame, the Dynamic Mesh technique was used, where the entire mesh moves with the velocity imposed by the orbital shaker, $\vec{U}$. The motion of the moving well was defined using an external $\mathrm{C}++$ user-defined function (UDF) linked to FLUENT. In this frame of reference, the force vector is:

$$
\vec{F}=\vec{g}
$$

The relative frame of reference is non-inertial and translating with the speed imposed by the orbital shaker such that the walls have zero-velocity relative to the frame. The influence of the plate motion is introduced through additional (pseudo-) force terms ${ }^{33}$ such that the force vector can be stated as:

$$
\vec{F}=\vec{g}-\frac{d \vec{U}}{d t}-\frac{d \vec{\omega}}{d t} \times \vec{R}_{\mathrm{p}}-2 \vec{\omega} \times \vec{v}_{\mathrm{rel}}-\vec{\omega} \times\left(\vec{\omega} \times \vec{R}_{\mathrm{p}}\right)
$$

where $\vec{R}_{\mathrm{p}}$ is the position vector from the origin in the moving frame and $\omega$ is the angular velocity of the rotation about the vertical axis of the reference frame. $d \vec{U} / d t, d \vec{\omega} / d t, 2 \vec{\omega} \times \vec{v}_{\text {rel }}$, and $\vec{\omega} \times\left(\vec{\omega} \times \vec{R}_{\mathrm{p}}\right)$ are the 
acceleration of the moving reference frame, the angular acceleration effect, Coriolis and centripetal acceleration, respectively. In orbital motion $\vec{\omega}=0$ and the last three terms in $\vec{F}$ vanish.

Both methods were implemented to verify the validity of the original assumptions. While the simulation results were in agreement within the numerical accuracy, it was found that the convergence rate was much faster and a coarser grid was sufficient when using the Dynamic Mesh technique. The time steps and grids needed in the moving frame of reference were approximately $6-8$ and 2 times finer, respectively. All results are reported in the relative coordinate frame with origin at the center of the well.

Since the motion of well of the culture plate is identical, simulations were conducted for a single well. The numerical grids were generated in GAMBIT. An example is shown in Fig. 1b. The grids generated in this geometry make it possible to refine the grids near the solid boundaries optimally: $90 \%$ of the total grids had a skewness of less than 0.2 and none of the cells had high skewness. ${ }^{9}$ The resolution of grids was increased at the walls with a geometric expansion coefficient of 1.04. Grid sensitivity was assessed by nominally doubling the grid density in successive simulations. The wall shear stress was found to be the most sensitive parameter to the change of grid density. The changes in shear stress were negligible between grids of 64,000 and 121,500 cells using dynamic mesh techniques. All results are reported for the geometry with 64,000 grids and the relative coordinate system $(x, y, z)$.

\section{WALL SHEAR STRESS MEASUREMENT USING MICRO SENSOR}

The magnitudes of the instantaneous wall shear stress components at the bottom of three modified Plexiglas wells were measured with a non-intrusive, optical MicroS sensor (Measurement Science Enterprise). The sensor is flush-mounted with the bottom surface of the well through a sealed hole as shown in Fig. 2. Radial and tangential components were
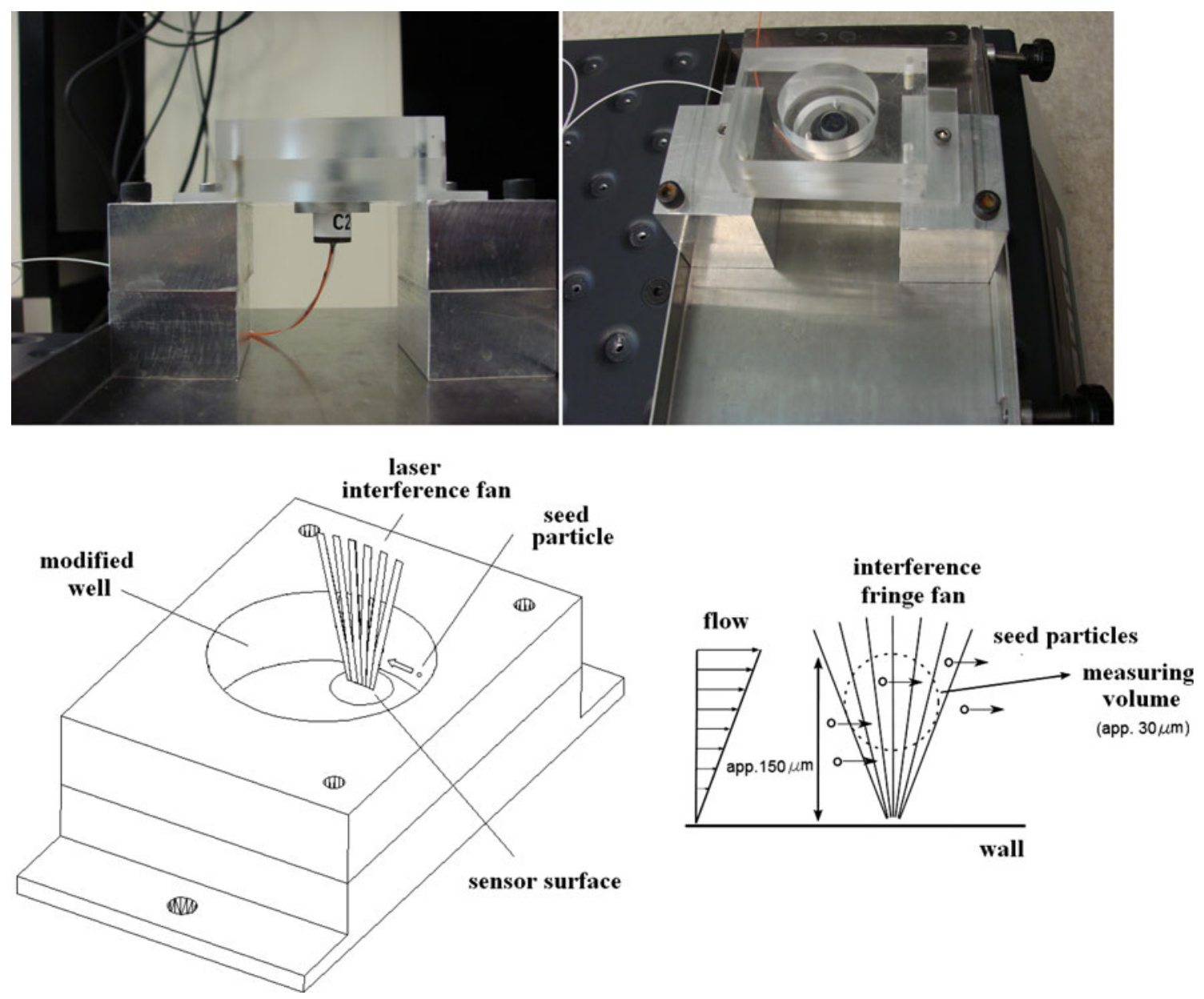

FIGURE 2. Shear rate sensor mounted flush with the well surface and schematic of sensor. 
measured independently at radial locations of 1,6 , and $12 \mathrm{~mm}$ from the well center. The sensor operates based on a modified Laser Doppler Anemometry technique. As shown schematically in Fig. 2, a linearly diverging interference fringe pattern is produced when a laser beam is incident on two closely spaced parallel slits. Approximating the velocity profile to be linear close to the wall (approximately within $150 \mu \mathrm{m}$ ), the frequency of the scattered light from seed particles in the direct vicinity of the wall is then independent of particle's position and directly related to the wall shear stress via $\left|\tau_{\mathrm{w}}\right|=\mu f_{\mathrm{d}} C$, where $\mu$ is the fluid viscosity, $f_{\mathrm{d}}$ is the Doppler frequency, and $C$ is a sensor characteristic (0.0526 for water). Shear stress magnitudes as low as $0.013 \mathrm{~Pa}$ can be measured with this device. The lower limit of the measurable wall shear stress is dictated by the high pass filter settings required for signal processing. To verify the accuracy of the sensor, it was tested in a laminar duct flow with known theoretical wall shear rate. The accuracy was within $\pm 10 \%$ of the instantaneous local wall shear rate. Silicon carbide (SiC) particles with mean diameter of $8 \mu \mathrm{m}$ were used to seed the flow. Data rate and signal-to-noise ration were higher than $15 \mathrm{~Hz}$ and $5 \mathrm{~dB}$, respectively. Raw and filtered signals were monitored on an oscilloscope. The band-pass filters were set such to optimize the signal-to-noise ratio and data rate. Details of sensor operation have been described in more detail by Sattari $^{26}$ and Wilson et al. ${ }^{34}$

\section{PLANAR FLOW FIELD MEASUREMENT USING PARTICLE IMAGE VELOCIMETRY}

The planar velocity field in a horizontal plane $2 \mathrm{~mm}$ from the bottom surface of the well was measured with a LaVision high frame-rate particle imaging Velocimeter (HFPIV). For this purpose a single well was manufactured from Plexiglas with the dimensions of the actual wells in culture plates. The bottom surface of the well was painted black to reduce reflections. A $527 \mathrm{~nm}, 20 \mathrm{~mJ} /$ pulse Photonics Industries Nd-YLF pulsed laser provided a 1-mm-thick laser sheet with a $12^{\circ}$ light fan. A HighSpeedStar camera $(1024 \times 1024$ pixels) was used with a $50 \mathrm{~mm}$ Nikkor macrolens objective to capture images in the single-frame (cinematographic) mode at $1000 \mathrm{~Hz}$ sampling rate. Interrogation windows of $16 \times 16$ pixel with $50 \%$ overlap resulted in a spatial resolution of $3.0 \mathrm{~mm}$ and a vector separation of $1.5 \mathrm{~mm}$. The flow was seeded using Silicon Carbide particles with $8 \mu \mathrm{m}$ mean diameter. A schematic of the HFPIV experimental setup is presented in Fig. 3.

To analyze the velocity vector field inside the well, the circular inner boundary of the well was detected from the recorded snapshots. Since the position of the camera was fixed, the translational velocity of the shaker was subtracted from the raw measured velocity field to obtain velocities in the frame of reference of the moving well. Averages of the velocity field at a given
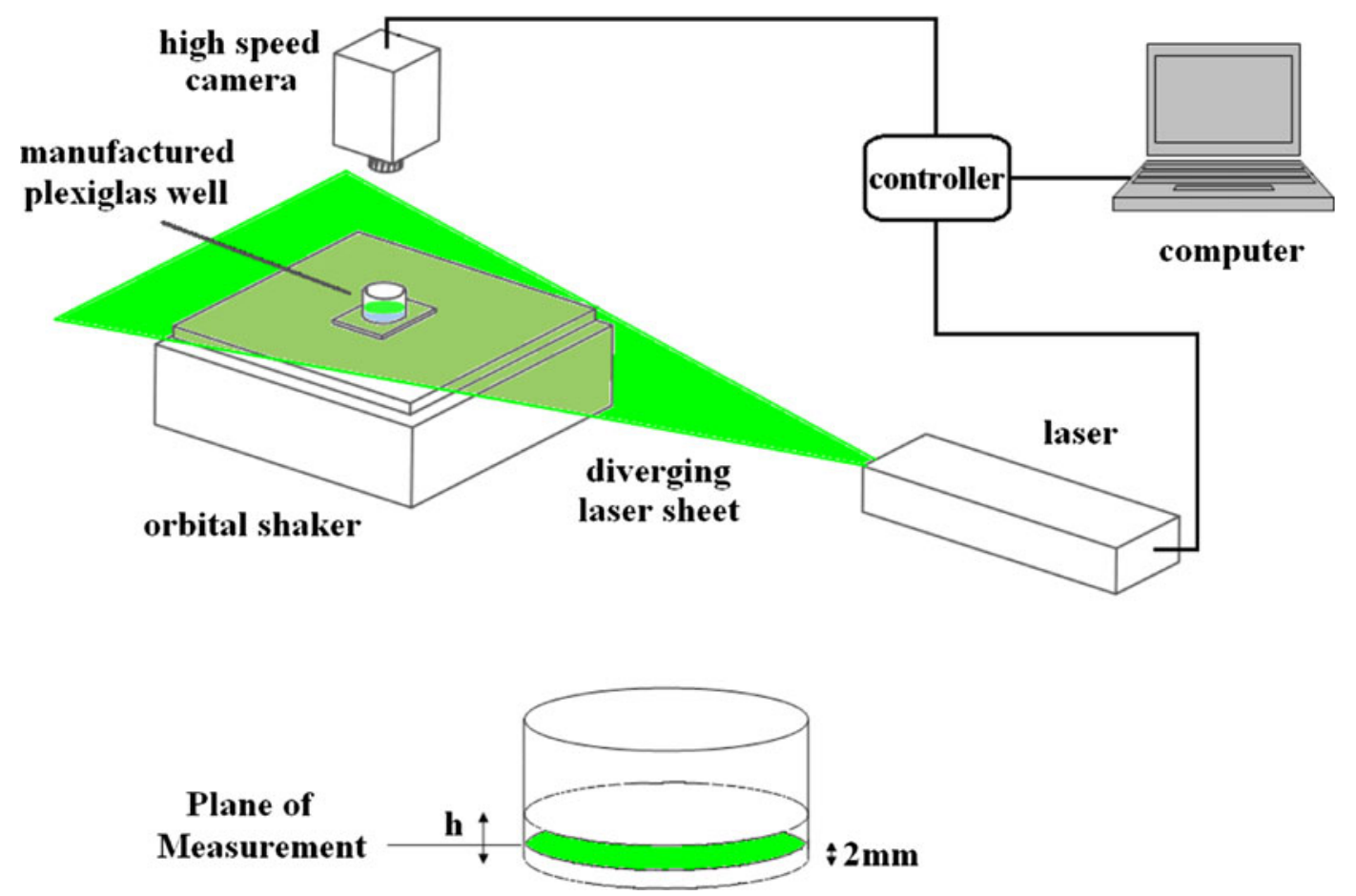

FIGURE 3. Schematic of experimental setup for High Frame-rate Particle Imaging Velocimetry (HFPIV). 
phase were calculated over approximately 10 cycles. The same pattern repeats with a pure rotation at other times within a cycle.

\section{FREE-SURFACE VISUALIZATION}

The motion of the free-surface was captured using a SONY model DSC-H5 digital camera mounted on the shaker and translated together with the well. The recording rate of the camera was 24 frames-per-second and hence approximately 14 images within a cycle could be captured at the shaker speed of $100 \mathrm{rpm}$. Food coloring was added to the fluid (water) for better picture contrast.

\section{RESULTS AND DISCUSSIONS}

\section{Model Validation}

The flow patterns inside a well undergoing twodimensional orbital motion in a plane $(X Z)$ perpendicular to gravity are investigated using CFD to understand how the wall shear stress patterns arise along the bottom face of the well. Results are compared for two rotational speeds $(\Omega=100$ and $200 \mathrm{rpm}$ ) and two liquid fill volumes (2 and $4 \mathrm{~mL}$ ). The validity of the numerical simulations is verified by comparison to experimental observations.

The magnitude of the mean radial and tangential wall shear stress components measured at three radial locations relative to the center of the well $(r=1,6$, and $12 \mathrm{~mm}$ ) agreed within experimental uncertainty with those predicted by CFD. Sample comparisons of instantaneous magnitudes at two locations are provided in Fig. 4. In this figure, spectra obtained from the shear rate sensor are also shown. The peak power occurs at the frequency corresponding to the orbital shaker forcing frequency, while the lower-power secondary peaks correspond to harmonics. Since the sensor measures relative to the moving plate, these results indicate that the shear stress field is moving relative to the plate. As will be shown below, this is consistent with the observation that the entire flow field rotates relative to the well at the forcing frequency.

HFPIV measurements were made in a plane $2 \mathrm{~mm}$ above and parallel to the bottom face of the well.
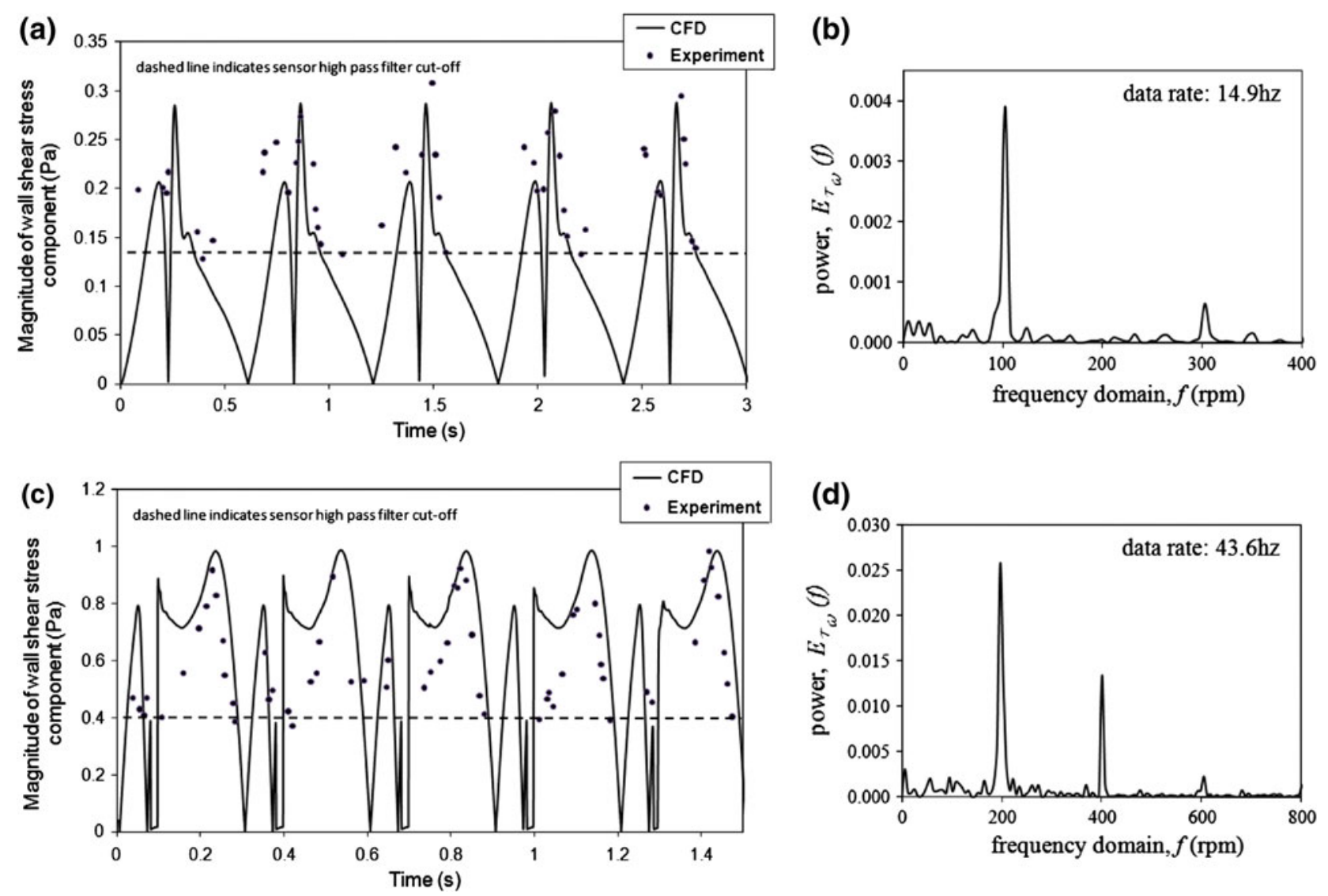

FIGURE 4. Comparison of the predicted and measured tangential wall shear stress magnitude as a function of time and their power spectrum for (a) and (b) $100 \mathrm{rpm}, 4 \mathrm{~mL}$ at $(r=12 \mathrm{~mm})$; (c) and (d) $200 \mathrm{rpm}, 4 \mathrm{~mL}(r=6 \mathrm{~mm})$. Dashed line indicates the highpass filter limit for the optical shear sensor. Note that time $t=t_{0}$ is arbitrary. 
Planar flow field from PIV measurements are compared with CFD results in Fig. 5 for one of four studied cases, i.e., $100 \mathrm{rpm}$ and $4 \mathrm{~mL}$ fluid volume. The dashed circle indicates the well boundaries. Close to the side wall (less than $5 \mathrm{~mm}$ ) the velocity vectors could not be resolved due to reflections. Sample profiles of the $z$-component velocity profiles along $x=0$ and $z=0$ are also shown. Instantaneous PIV results show a good agreement between experiments and CFD results both qualitatively (flow pattern) and quantitatively (velocity magnitudes) as shown in Fig. 5.

The numerically predicted and experimentally observed free surface motion also agrees well. Sample comparisons for $\Omega=100 \mathrm{rpm}$ and $4 \mathrm{~mL}$ are at different times of the shaker cycle are shown in Fig. 6. The free surface is characterized by a traveling wave undergoing solid body rotation at the rate of $\Omega$ about the center axis of the well. The shape and elevation of the predicted and observed free-surface agree well, validating the underlying assumptions (with respect to surface tension) and suggesting an approach for modeling of the free-surface dynamics. Generally, the shape of the free-surface resembles an inclined horseshoe over the elevated fluid region. As will be discussed later, regions of low liquid level, or troughs, are associated with a thin film of rapidly moving fluid. The high fluid level regions lagging the leading wave crest give rise to a complex system of counter-rotating vortices. In addition to the wall shear stress on the bottom wall, the shape of the free surface is also an important consideration in interpreting biological results. For example, at $\Omega=100 \mathrm{rpm}$, the fluid covers the entire bottom face of the well for both fill volumes. At $\Omega=200 \mathrm{rpm}$, a small portion of the well bottom face is exposed near the distal corners in the trough of the horseshoe. This exposure to air during portion of the cycle is expected to affect the culture area (e.g., biofilm growth) in these regions. (a)

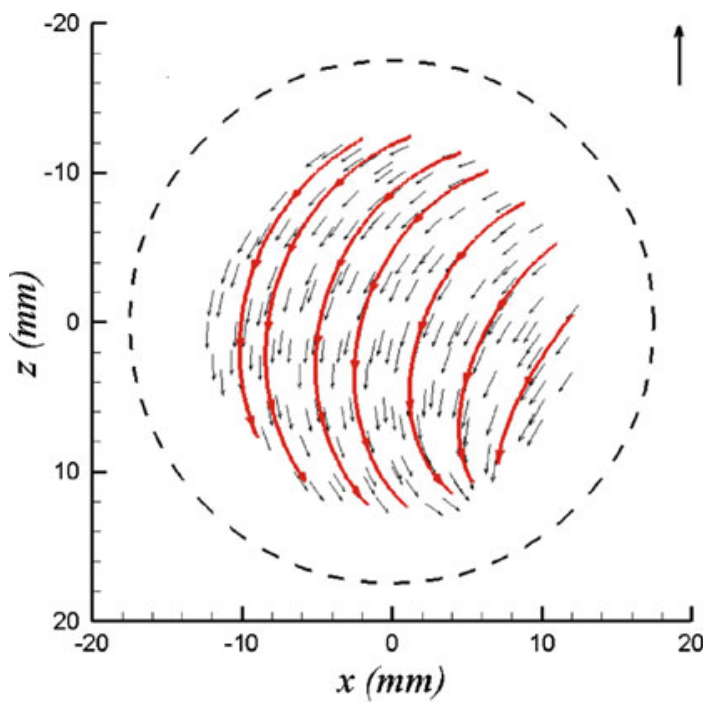

(c)

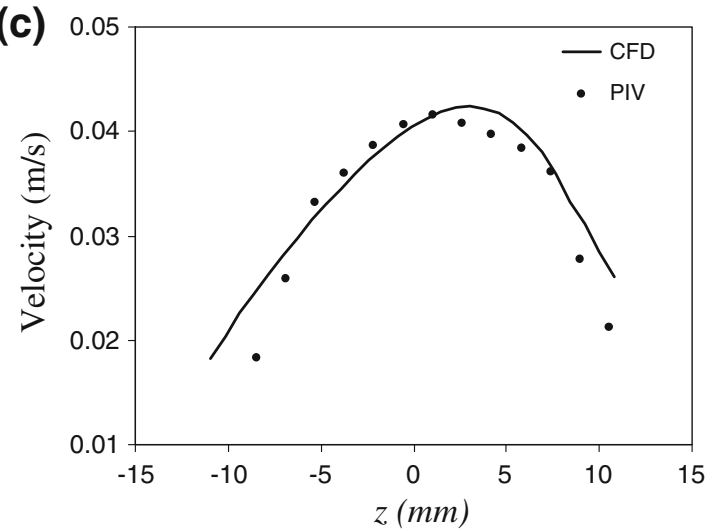

(b)

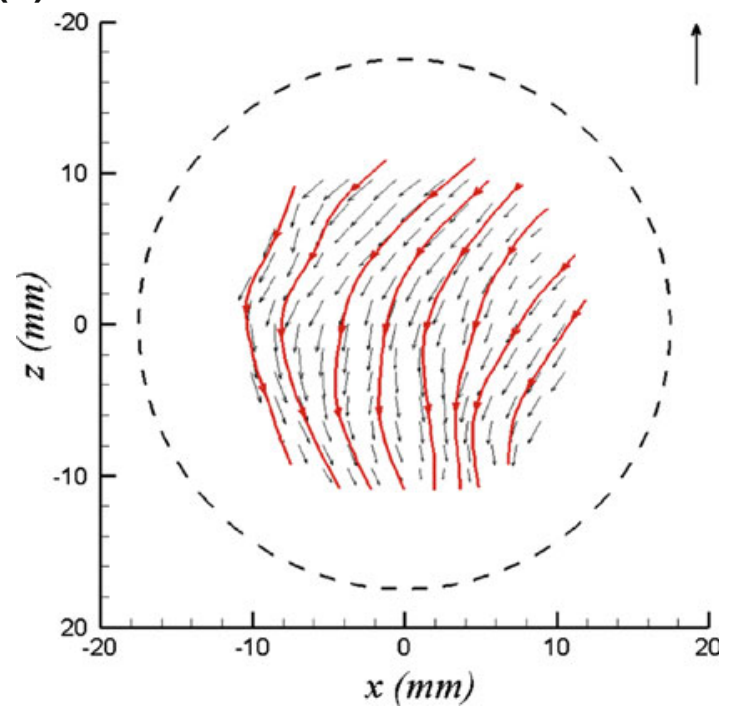

(d)

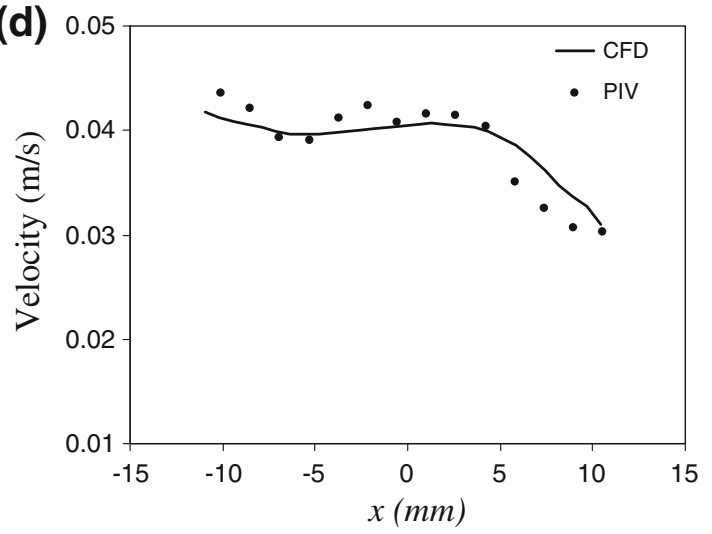

FIGURE 5. Comparison of CFD and HFPIV velocity vector snapshot at $y=2 \mathrm{~mm}$ (a) CFD; (b) PIV; (c) comparison of $z$-component of velocity at $x=0$; (d) comparison of $z$-component of velocity at $z=0$. The reference vector magnitude is $0.1 \mathrm{~m} / \mathrm{s}$. 

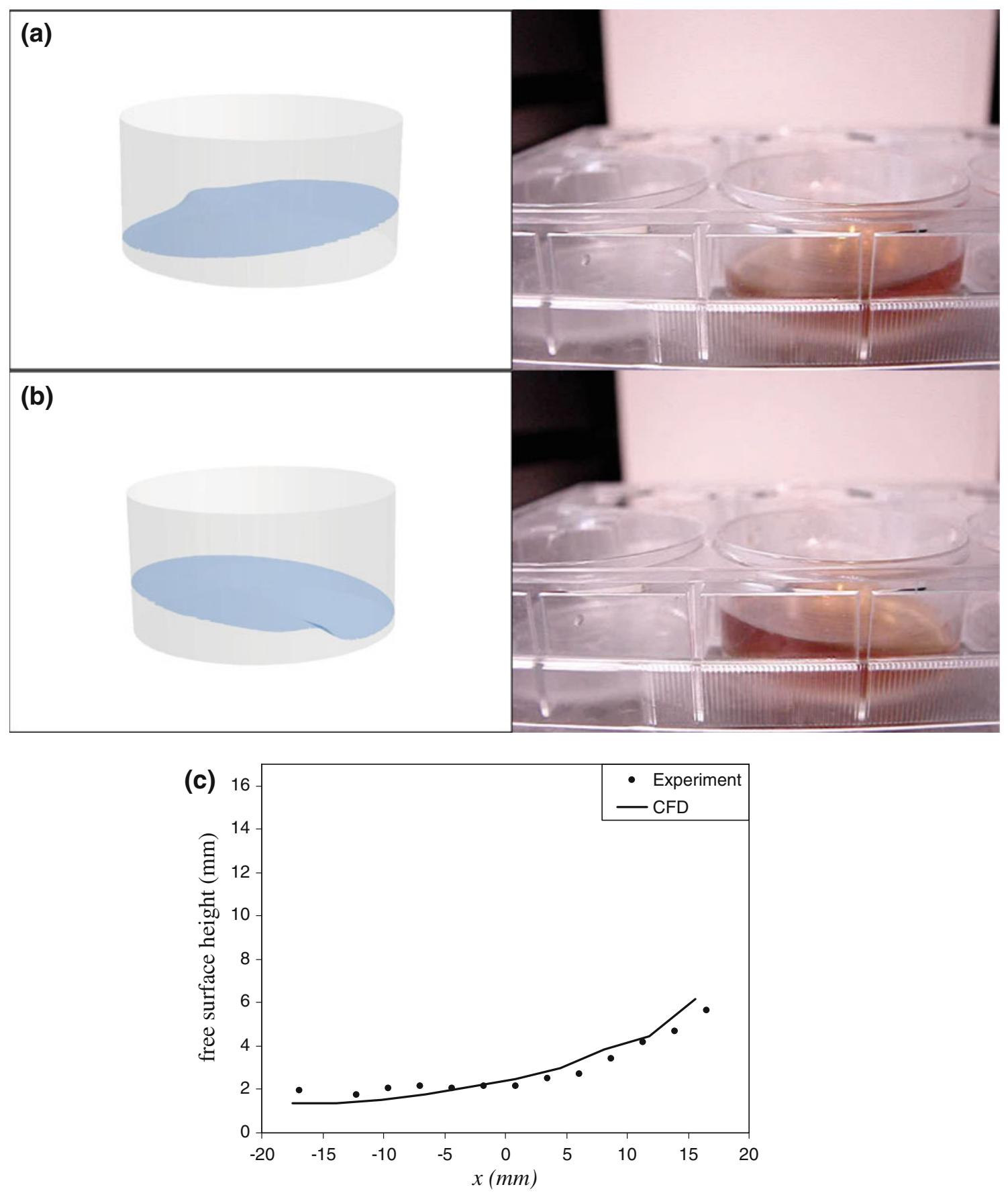

FIGURE 6. Visualization of free surface at $100 \mathrm{rpm}$ and $4 \mathrm{~mL}$ at (a) $t=t_{0}$; (b) $t=t_{0}+T / 2$; (c) comparison of CFD and experiment. $T$ is the period of oscillation.

\section{Prediction of Free Surface Level}

The shape of the free-surface and motion of the horseshoe liquid torus, generally located in high liquid elevation regions, can be approximated using a simplified heuristic argument. Based on the observation that the torus undergoes a rigid rotation about the center axis of the well, it is advantageous to consider the problem in a translating, non-inertial reference frame with origin fixed at the well center. Assuming that viscous effects are restricted to the immediate vicinity of the solid boundaries, the net effect of the wall shear stress is to induce the solid rotation about the well center such that the relative tangential component of velocity (averaged over a vertical line in the liquid layer) is approximated simply as:

$$
\bar{u}_{\theta}=\Omega r
$$


where $r$ is the radius from the well center. Assuming that the vertical component of velocity, which is bounded by the well and free surfaces, is small compared to $\bar{u}_{\theta}$, it is found on grounds of continuity that the radial component of the fluid velocity must also be small. Hence, in this reference frame, the transient terms approximately vanish and the shape of the free surface is governed by the pressure distribution, $P$, which can be described by a simplified form of the momentum equations in the vertical, $y$, and radial directions, respectively:

$$
\begin{gathered}
0=-\frac{\partial P}{\partial y}-\rho g \\
-\rho \frac{\bar{u}_{\theta}^{2}}{r}=-\frac{\partial P}{\partial r}-\rho a_{\mathrm{rel}}
\end{gathered}
$$

where $\rho$ is the liquid density and $a_{\text {rel }}$ the acceleration of the translating reference frame:

$$
a_{\text {rel }}=-R_{\mathrm{g}} \Omega^{2}[\cos (\theta-\phi)+\sin (\theta-\phi)]
$$

where $\theta=\Omega t$ corresponds to the angular orientation of the acceleration vector at an arbitrary time $t$. The motion of the fluid over an oscillating well lags the motion of the well bottom wall (cf. Stokes' second problem ${ }^{27}$ ) by a phase $\phi$, which is determined empirically.

An expression for the pressure field is obtained upon integration of the momentum equations:

$P=\rho \frac{\Omega^{2} r^{2}}{2}-\rho g y+\rho R_{\mathrm{g}} \Omega^{2}[r \cos (\theta-\phi)+r \sin (\theta-\phi)]+C_{0}$

where $C_{0}$ is a constant of integration. Denoting the free surface location, where the gauge pressure is zero by definition, by $y=h$ :

$h=\frac{\Omega^{2}}{g}\left[R_{\mathrm{g}}(r \cos (\theta-\phi)+r \sin (\theta-\phi)+R)+\frac{r^{2}}{2}\right]+C$

where $C$ is a constant of integration.

Further insight can be gained by recasting this relationship in terms of a Cartesian coordinate system $\left(x^{\prime}, z^{\prime}\right)$ with origin at the center of the well and aligned with the direction of $a_{\text {rel }}$, which is equivalent to rotating the coordinate system through an angle of $\theta-\phi$ such that the expression for the free-surface becomes:

$$
h=\frac{\Omega^{2}}{g}\left[R_{\mathrm{g}}\left(x^{\prime}+R\right)+\frac{\left(x^{\prime 2}+z^{\prime 2}\right)}{2}\right]+C
$$

This relationship can be further modified by completing the squares to obtain:

$$
h=\frac{\Omega^{2}}{2 g}\left[\left(x^{\prime}+R_{\mathrm{g}}\right)^{2}+z^{\prime 2}-R_{\mathrm{g}}^{2}+2 R R_{\mathrm{g}}\right]+C
$$

Hence, the free-surface describes a parabola with focus at $x^{\prime}=-R_{\mathrm{g}}, z^{\prime}=0$ and the focus rotates about the center of the well at a rate $\Omega$.

The constant of integration, $C$, is determined by requiring that the volume enclosed under the freesurface equal the total volume of liquid, $V$, present in the well. If the free-surface does not intersect the bottom surface of the well, this condition yields:

$$
C=\frac{V}{\pi R^{2}}-\frac{\Omega^{2}}{g} R\left(R_{\mathrm{g}}+\frac{R}{4}\right)
$$

If the free-surface intersects the well bottom, by virtue of parabolic distribution described above, the contact point will describe a circle of radius $R_{\mathrm{a}}$ about $x^{\prime}=-R_{\mathrm{g}}, z^{\prime}=0$. Thus, the volume of fluid under the free-surface (and above the plate bottom surface) is given by:

$$
V=\frac{\pi \Omega^{2}}{4 g}\left[2 R^{2}\left(\frac{R^{2}}{2}+R_{\mathrm{g}}^{2}\right)-2 R_{\mathrm{a}}^{2}+R_{\mathrm{a}}^{4}\right]
$$

and, the integration constant is given by:

$$
C=\frac{\Omega^{2}}{4 g R^{2}}\left[R_{\mathrm{a}}^{4}-2 R_{\mathrm{a}}^{2}\right]
$$

where

$$
\begin{aligned}
& R_{\mathrm{a}}^{2}=R^{2}-\sqrt{R^{4}-B} \\
& B=2 R^{2}\left(\frac{R^{2}}{2}+R_{\mathrm{g}}^{2}\right)-\frac{4 g}{\pi \Omega^{2}} V \quad R_{\mathrm{a}}<R+R_{\mathrm{g}}
\end{aligned}
$$

The comparison of the numerically predicted free surface with theoretical equations has been summarized in Fig. 13. The results are in excellent agreement, accurately capturing: the locations where the bottom of the well is exposed (important for biological tests); the bulk motion of the fluid around the well; the curvature of the free surface (and thus the main surface dynamics) as well as the general sloshing behavior. This analytical form conveniently expresses the free surface behavior in closed form (all inputs are determined a priori by the user) (Fig. 7).

\section{Topology of Flow Field and Wall Shear Stress Distribution}

For an arbitrary, fixed point on the well surface, the shear stress fluctuates about the mean with a frequency of $\Omega$, as determined from the power spectra of the fluctuating component. Examples of this behavior are shown for two cases in Fig. 4. This periodic behavior is 

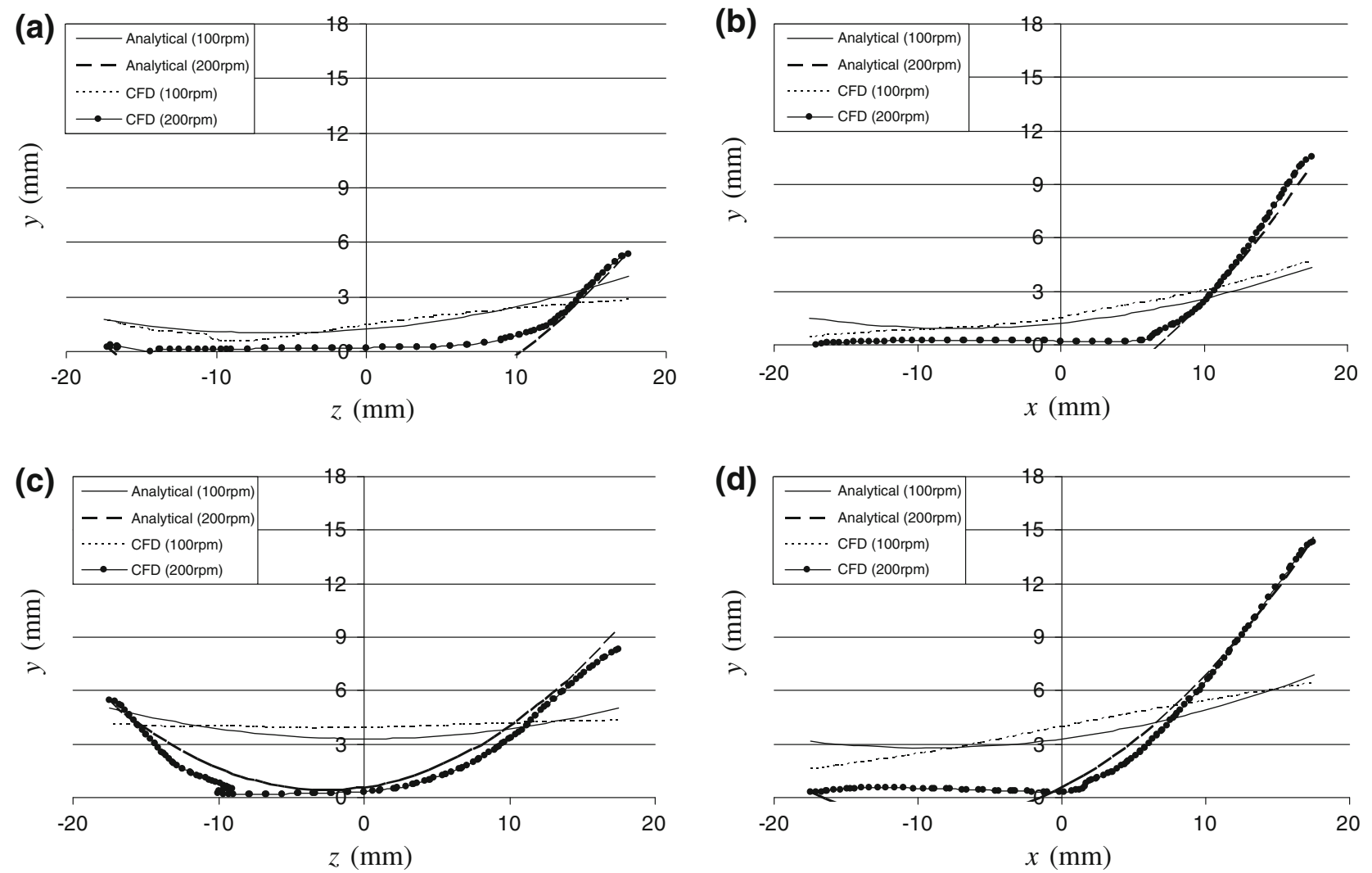

FIGURE 7. Comparison of the numerically predicted free surface with theoretical equations (a) $2 \mathrm{~mL}, x=0$; (b) $2 \mathrm{~mL}, z=0$; (c) $4 \mathrm{~mL}, x=0$; (d) $4 \mathrm{~mL}, z=0$.

consistent with the observed solid body rotation of the free surface about the well center.

An overview of the mean wall shear stress field on the bottom of the well is given in terms of the radial distribution in Fig. 8. The mean and fluctuating fields are predicted to be axisymmetric about the center of the well, which is consistent with the observed surface motion. Together, these observations suggest a solid body rotation of the fluid field about the center of the well.

The mean wall shear stress field is shown in Fig. 8a. Generally, the magnitude of the wall shear stress, $\tau_{\mathrm{w}}$, increases as the rotational rate $\Omega$ increases. The trends observed in the radial distribution change as a function of $\Omega$ and the liquid fill volume.

A two-dimensional extension of Stokes' second problem ${ }^{27}$ ) for an infinite, immersed plate undergoing circular (orbital) motion has been used extensively ${ }^{7,16,17}$ to estimate the magnitude of the wall shear stress, $\tau_{\mathrm{w}}$, at the bottom of the plate. For a constant rotational rate $\Omega$, Stokes' approximation yields that the magnitude of the wall shear stress is constant over the entire plate surface and has the value:

$$
\tau_{\mathrm{w}}=R_{\mathrm{g}} \Omega^{3 / 2} \sqrt{\rho \mu}
$$

This approach has been used ad hoc, ${ }^{3,7,16,17}$ but its applicability warrants further consideration since it ignores the influence of the well walls and finite volume fill.

At $\Omega=100 \mathrm{rpm}$, the time averaged wall shear stress magnitude $\tau_{\mathrm{w}}$ is nearly constant over the entire well bottom, but the absolute level changes with volume fill. Whereas the Stokes' approximation predicts a value of $0.32 \mathrm{~Pa}$ for the present test conditions, the observed values in Fig. 8a are 0.28 and $0.15 \mathrm{~Pa}$, for 2 and $4 \mathrm{~mL}$ volume fill, respectively. At $\Omega=200 \mathrm{rpm}, \tau_{\mathrm{w}}$ is approximately constant only near the center $(r<2 \mathrm{~mm})$, where the value is close to $0.83 \mathrm{~Pa}$ as compared to $0.91 \mathrm{~Pa}$ as predicted from Stokes' approximation. The average shear stress magnitude over the bottom of the well at $\Omega=200 \mathrm{rpm}$ is 0.87 and $1.02 \mathrm{~Pa}$ for 2 and $4 \mathrm{~mL}$ volume fill, respectively. For this higher rotation rate, the influence of the volume fill is increasingly observed as the container distal wall is approached, such that the wall shear stress on the well bottom may not be suitably represented by a single, average value.

These results suggest that Stokes' approximation can lead to erroneous conclusions when comparing 
(a)

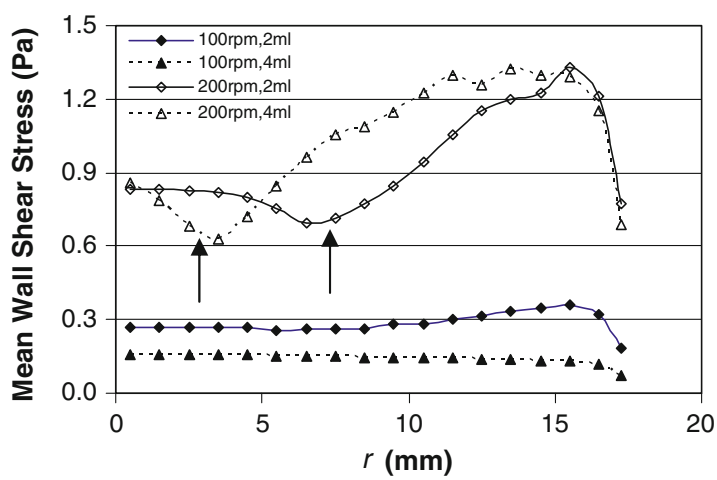

(b)

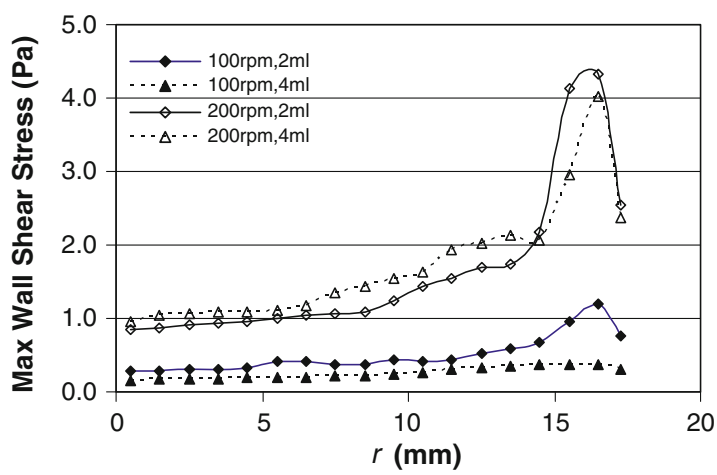

(c)

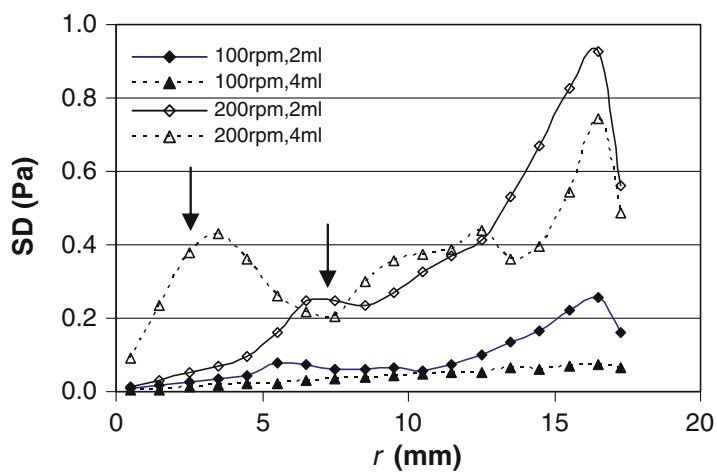

FIGURE 8. Summary of the radial distribution of the wall shear stress magnitude $(\mathrm{Pa})$ as a function of the radial location: (a) mean levels; (b) maximum shear magnitude; and (c) standard deviation of the shear stress fluctuation.

results at different $\Omega$ or when the influence of the volume fill is neglected. Note that in this work, the volume fills are typical of most biological experiments. Moreover, $\tau_{\mathrm{w}}$ is not constant (in time) on the well bottom as can be seen from the standard deviation of the fluctuations shown in Fig. 8c. In fact, Fig. 8a presents the time averaged of mean wall shear stress magnitude $(\mathrm{Pa})$ as a function of the radial location. The fluctuation distribution changes with both rotational rate and volume fill, indicating an important influence of the dynamics. ${ }^{15}$ Thus, in considering results from biological experiments and especially for comparison purposes, further attention should be given to the changes in the flow pattern as a function of the operating parameters. A description of the flow field and its topology follows, from which several features observed in the distributions of the Fig. 8 are elucidated.

Like the motion of the free surface, the entire flow field undergoes a solid body rotation about the well center axis. This behavior is confirmed by inspection of the shear stress field at different time points in the shaker cycle and thus a view of the flow field at a single time point is sufficient. In Fig. 9, the wall shear stress magnitude distribution on the bottom face of the well is shown for all four cases investigated at an arbitrary time $\left(t=t_{0}\right)$ during the orbital cycle. The corresponding elevation contours of the liquid levels and 3D structure of the free surfaces are shown in Figs. 10 and 11, respectively. In all cases, the shear stress field rotates counter-clockwise about the well center axis in the direction and at the rotational rate of the orbital shaker. The surface-stress streamlines (tangential to the surface shear stress vector at all points) are superimposed to assist in the interpretation of the flow patterns in subsequent sections. The shear stress vector is oriented in the direction of the flow in the immediate proximity of the bottom surface. It will be noted that for any radial position, the same cyclical (generally not sinusoidal, e.g., Figs. 13b, 13c) fluctuation of the wall shear stress level is observed with a period corresponding to the shaker rotation frequency, albeit with a phase difference which is purely a linear function of the tangential location. Consequently, the mean flow and wall stress fields are axisymmetric, but the instantaneous flow field contains significant tangential gradients and thus differs fundamentally from a pure rotation.

The shear stress distribution can be related to the topology of the fluid flow structure. Consider first the simpler structure case $(\Omega=100 \mathrm{rpm}, 4 \mathrm{~mL})$. Inspection of the velocity vectors in the perpendicular planes shown in Fig. 12a, together with the surface stress and free surface height (Figs. 10a, 11a), shows that the bottom surface of the well is never exposed to air. A large (counter-clockwise rotating) semi-torus vortex characterizes the bulk of the liquid motion in the elevated fluid region. Generally, the flow is directed from high to low fluid elevation along the well bottom and the wall shear stress magnitude increases radially outward along this structure. The flow impinges in the distal corner accounting for the low wall shear stress levels observed in the region $(r \rightarrow \mathrm{R})$. The fluid motion is helical about the tangential axis of the torus, forcing fluid along the bottom surface from high liquid level locations to the trough region, giving rise to a convergent node (focus) in the corner region $(x \sim-15 \mathrm{~mm} ; z \sim-15 \mathrm{~mm})$ along the bottom surface. Along the bifurcation line originating at the node, the 
(a)
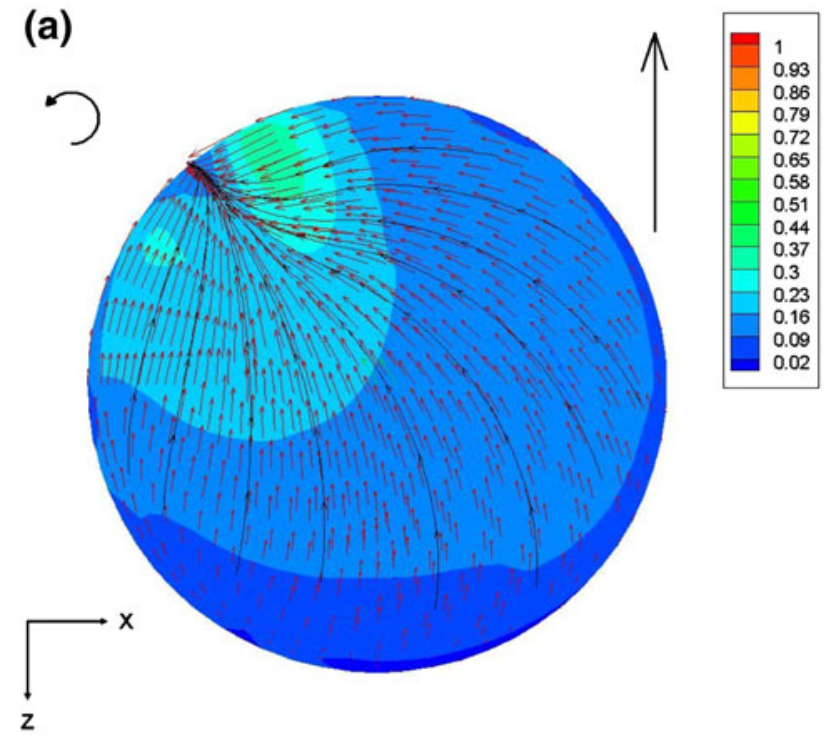

(c)
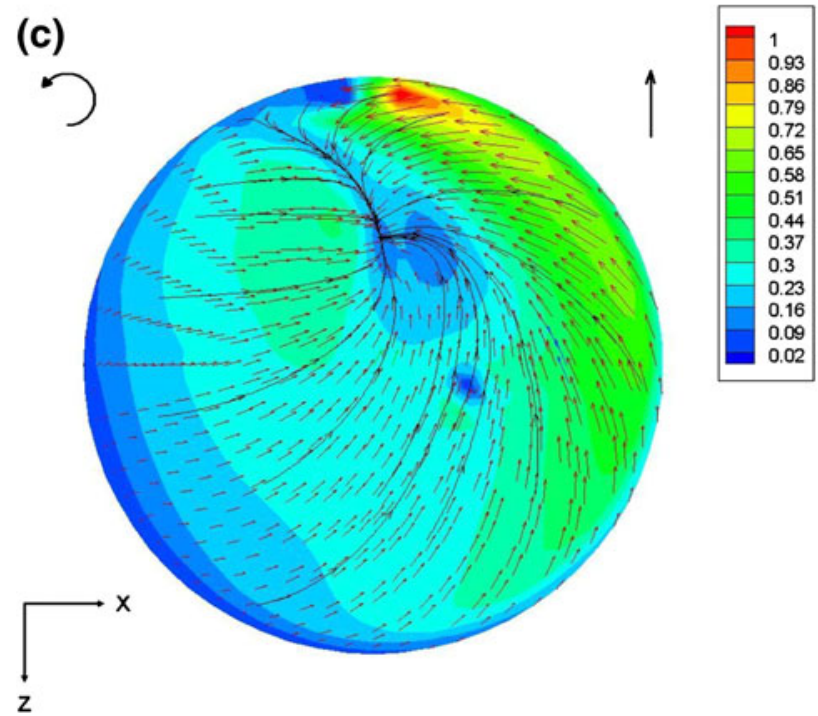

(b)

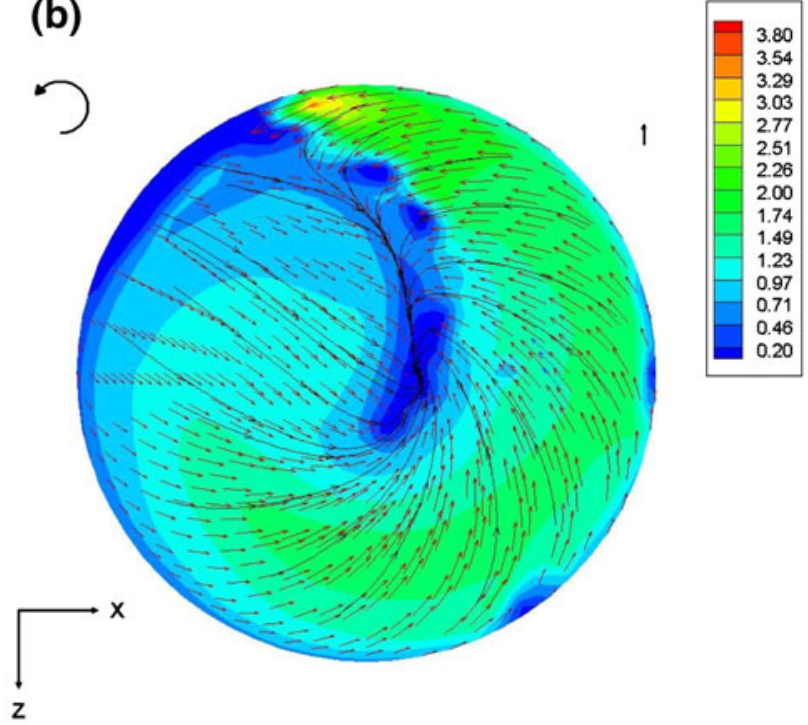

(d)

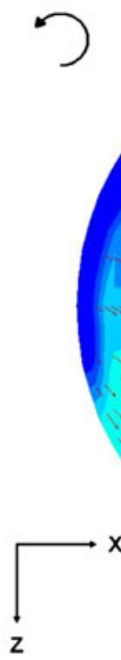

FIGURE 9. Contours of the magnitude of wall shear stress $(\mathrm{Pa})$ on the bottom face with surface "streamlines" superimposed at $t=t_{0}$ for (a) $100 \mathrm{rpm}, 4 \mathrm{~mL}$; (b) $200 \mathrm{rpm}, 4 \mathrm{~mL}$; (c) $100 \mathrm{rpm}, 2 \mathrm{~mL}$; and (d) $200 \mathrm{rpm}, 2 \mathrm{~mL}$. Curved arrow indicates sense of rotation. The units are in $\mathrm{Pa}$ and the reference vector magnitude is $1 \mathrm{~Pa}$.

tangential component of the shear stress changes sign and the node is located between the local magnitude maxima (a positive and a negative, see Fig. 9a).

These flow structures will cross any fixed radius on the plate during one cycle of rotation, which results in a cyclical fluctuating shear stress when observed at a fixed point as shown in Fig. 13. Near the plate center (Fig. 13a), the liquid motion is almost sinusoidal, resembling that over an oscillating plate. The wave form is gradually distorted by the rotation of the flow field as $r$ is increased (for example in Figs. 13b and 13c at the location $r=6 \mathrm{~mm}$ and $r=12 \mathrm{~mm}$ ). Together with Fig. 9a, a local magnitude maximum (positive sign) of the tangential shear stress occurs behind (lagging) the bifurcation line in the elevated liquid region.
This shear component decreases under the semi-torus (horseshoe) vortex and through the trough region, reaching a magnitude maximum (negative sign) directly ahead of the wave crest. The shear stress values jump abruptly across the bifurcation line, which coincides with a zero tangential stress line and a sudden flow reversal.

An increase in the rotational speed of the shaker causes approximately a 3-fold increase of the mean shear levels over the entire plate (Fig. 8a), but may result in up to a 10- to 12-fold increase in the maximum shear levels (Figs. 8b and 9b, mainly in the distal corners) and significant changes in the liquid level across the well (Fig. 10b). The horseshoe shape of the elevated liquid regions becomes more distinct. In the 

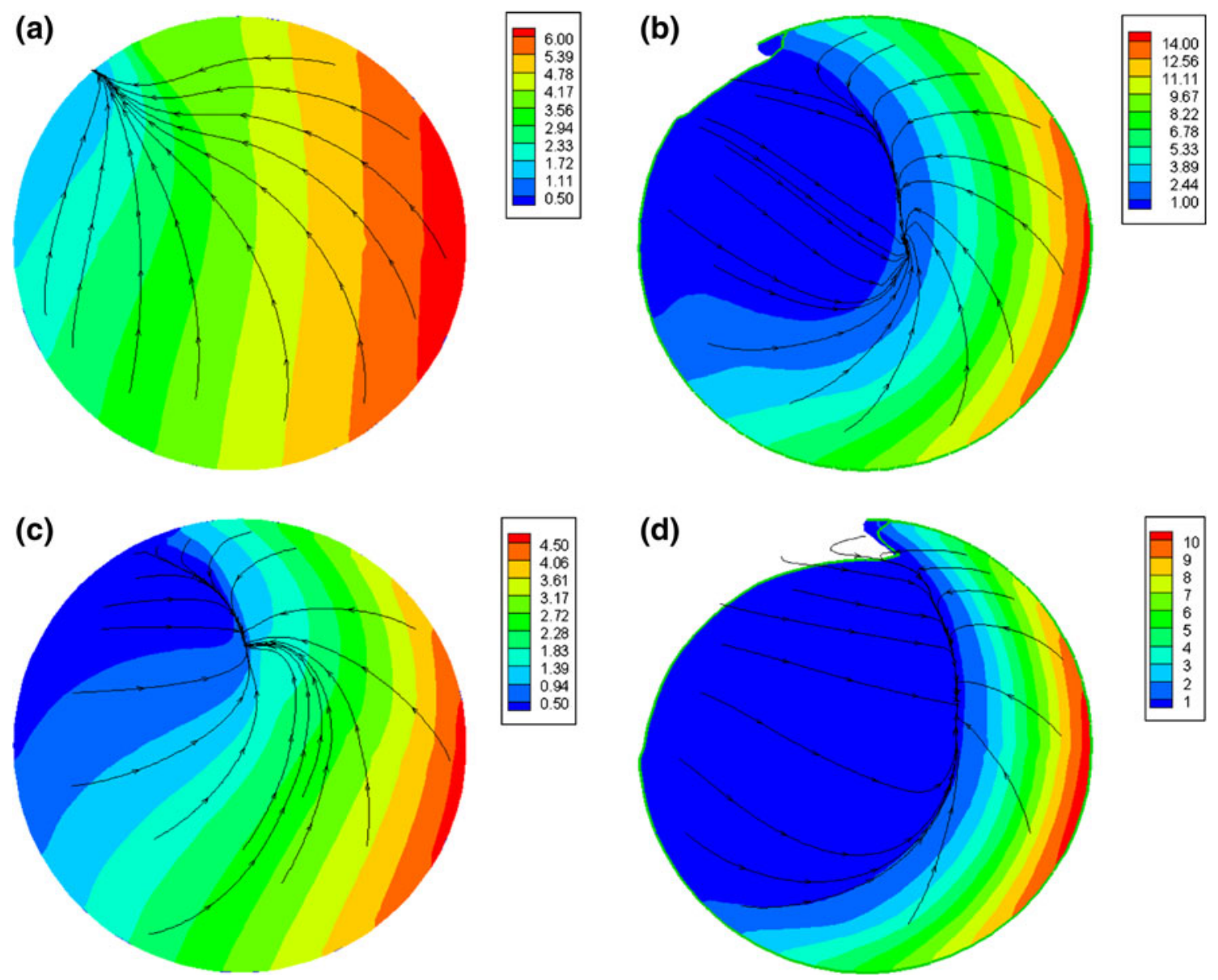

FIGURE 10. Elevation contours of the liquid level (free surface) at $t=t_{0}$ for (a) $100 \mathrm{rpm}, 4 \mathrm{~mL}$; (b) $200 \mathrm{rpm}, 4 \mathrm{~mL}$; (c) $100 \mathrm{rpm}$, $2 \mathrm{~mL}$; and (d) $200 \mathrm{rpm}, 2 \mathrm{~mL}$. The units are in mm. Curved arrow showed in the previous figure indicates sense of rotation.

well region surrounded by the horseshoe, a deep trough forms, resulting in a thin film of liquid moving rapidly away from the wall (contiguous with the open end of the horseshoe). A region exposed to air also appears near the vertical (distal) wall (Figs. 10b, 11b).

At the center of the well, the time series of the shear stress components again show a sinusoidal fluctuation. However, outside of the immediate vicinity of the center, the shear stress evolution resembles more that shown in Fig. 13c for $r=12 \mathrm{~mm}$. Generally, over much of the cycle, the bottom face is covered by the horseshoe structure and the tangential stress component is positive and changes little. The trailing edge of the wake is characterized by a rapid decrease of the shear, becoming negative in the thin film layer and reaching a magnitude maximum (negative sign) immediately ahead of the bifurcation line. The bifurcation line, similarly to the previous case, is found to lead the crest of the wave. The flow and tangential shear stress abruptly change direction across the bifurcation line.

Velocity vector plots in two perpendicular vertical planes are shown in Fig. 12b for the case $200 \mathrm{rpm}$,
$4 \mathrm{~mL}$. The curvature of the free surface is much more pronounced than in the previous case. The base of the trough consists mainly of a thin film of water moving rapidly from the distal region of the trough to the elevated liquid region. Inside the elevated liquid regions, typically two counter-rotating vortices are observed (see Fig. 12b). In the plane $z=0$, the flow near the bottom surface moves rapidly from the outer wall toward the plate center, where it meets the opposing thin film flow, deflecting the fluid radially outward below the free surface. The bifurcation line lags the leading wave edge slightly.

These vortex structures can also be observed in the plane $x=0$. On the trailing side of the wave, $z>0$, the flow along the well bottom is forced from the distal wall to the center, where it meets the opposing flow from the film layer, thus inducing a radially outward flow beneath the free-surface. In this plane, the secondary (counter-rotating) vortex in the distal corner below the free-surface is stronger and more easily recognized. This vortex induces a radially inward flow along the free-surface to about a radius of $r=10 \mathrm{~mm}$ $(z=10 \mathrm{~mm}$ in Fig. 12b) to encounter the radially 
(a)

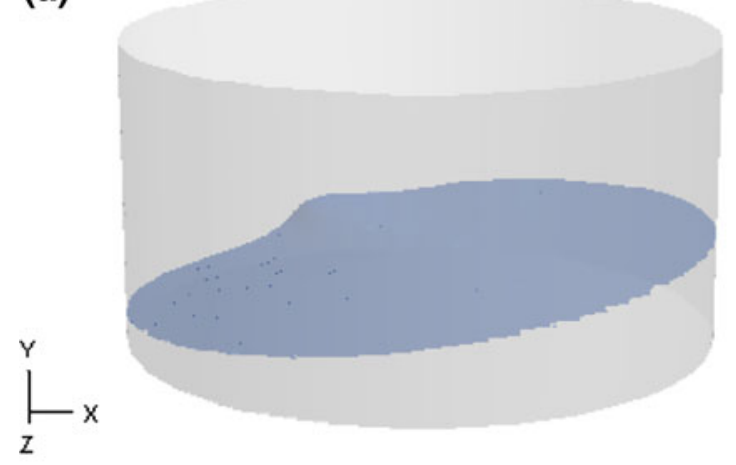

(c)

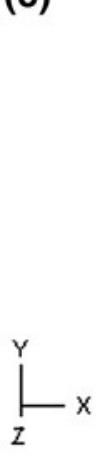

(b)
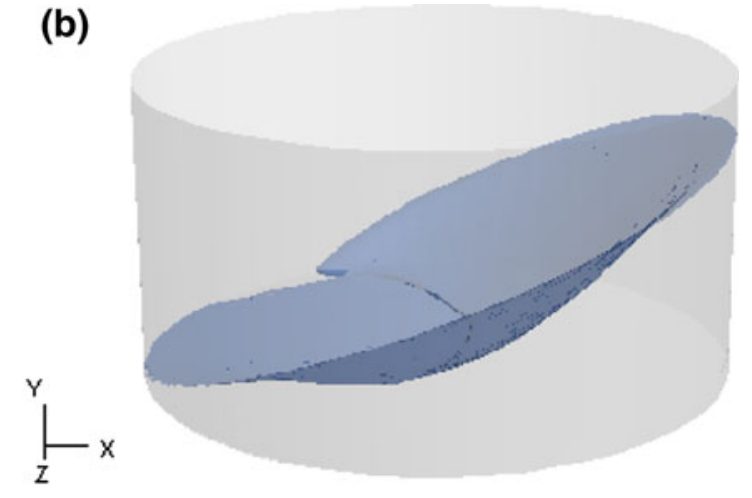

(d)

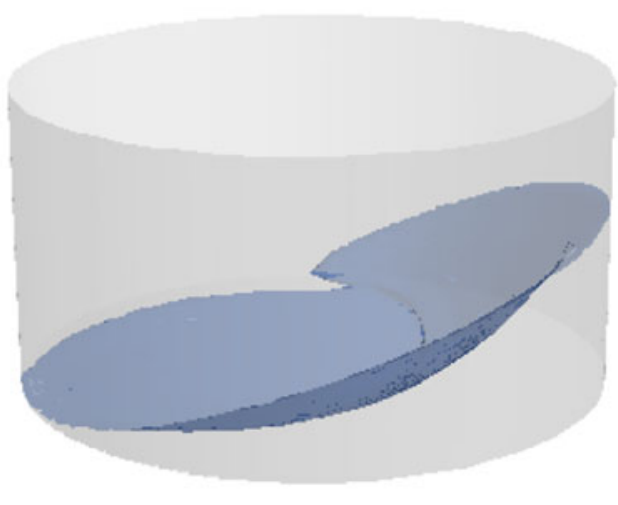

FIGURE 11. 3D structure of free surfaces at $t=t_{0}$ for (a) $100 \mathrm{rpm}, 4 \mathrm{~mL}$; (b) $200 \mathrm{rpm}, 4 \mathrm{~mL}$; (c) $100 \mathrm{rpm}, 2 \mathrm{~mL}$; and (d) $200 \mathrm{rpm}$, $2 \mathrm{~mL}$.

outward surface current, originating from the trough, and resulting in a sub-ducted stream. At the convergence point of these two streams, a change in curvature of the free-surface is observed.

On the leading side of the wave $(z<0)$, the flow along the bottom surface is radially inward through the elevated and thin film region. The elevated liquid region is dominated by a very strong recirculation which entrains fluid upward toward the free-surface and resulting in the leading wave crest. Figure $9 \mathrm{~b}$ shows that a converging (negative) bifurcation line slightly leads the crest region. The shear stress magnitude is small in the vicinity of the bifurcation line and reaches maxima below the core of the vortex and in the film region leading the bifurcation line. The radial component of the shear is negative in this region, while the tangential component of shear stress changes signs across the bifurcation line.

The flow and wall shear stress fields for the $2 \mathrm{~mL}$ cases are topologically similar to the corresponding cases at $4 \mathrm{~mL}$ quantitatively, however, some differences are observed. The same horseshoe pattern and presence of a bifurcation line can be recognized (Figs. 9c, 9d). However, due to the lower fluid levels, the trough regions are more pronounced and the thin film regions extend over a larger area. For the $200 \mathrm{rpm}, 2 \mathrm{~mL}$ case, the distal region of the plate bottom surface exposed to air during the cycle increases. These quantitative changes are reflected by sharper transitions between elevated liquid and thin film regions.

The forgoing discussion helps clarify the trends observed in the mean field as summarized in Fig. 8. The axisymmetry of the mean stress field results because the flow field is rotating relative to the center axis of the well. Note that the wall shear stress magnitude, rather than the components, was selected since it is expected that bacteria or mammalian cells will be more sensitive to the local magnitude of shear level and fluctuations than to the direction. The mean shear stress magnitude, $\tau_{\mathrm{w}}$, can be interpreted synonymously as either the time-average at a point, or tangentially averaged in space at constant radius for a fixed time point. As shown in Fig. 8a, $\tau_{\mathrm{w}}$, varies little across the plate for $\Omega=100 \mathrm{rpm}$, except close to the distal corners due to the secondary flow. In generally, regions of larger radial gradients are associated with regions of secondary flow structures. Doubling of the rotation rate to $\Omega=200 \mathrm{rpm}$ causes significantly larger radial gradients, which agrees with the appearance of significantly larger secondary flow regions. Thus, not surprisingly, in secondary flow regions the mean shear levels are not well predicted using stokes' plate approximation. Conversely, for the case $100 \mathrm{rpm}$, $2 \mathrm{~mL}$, the strength of the secondary motion is much 

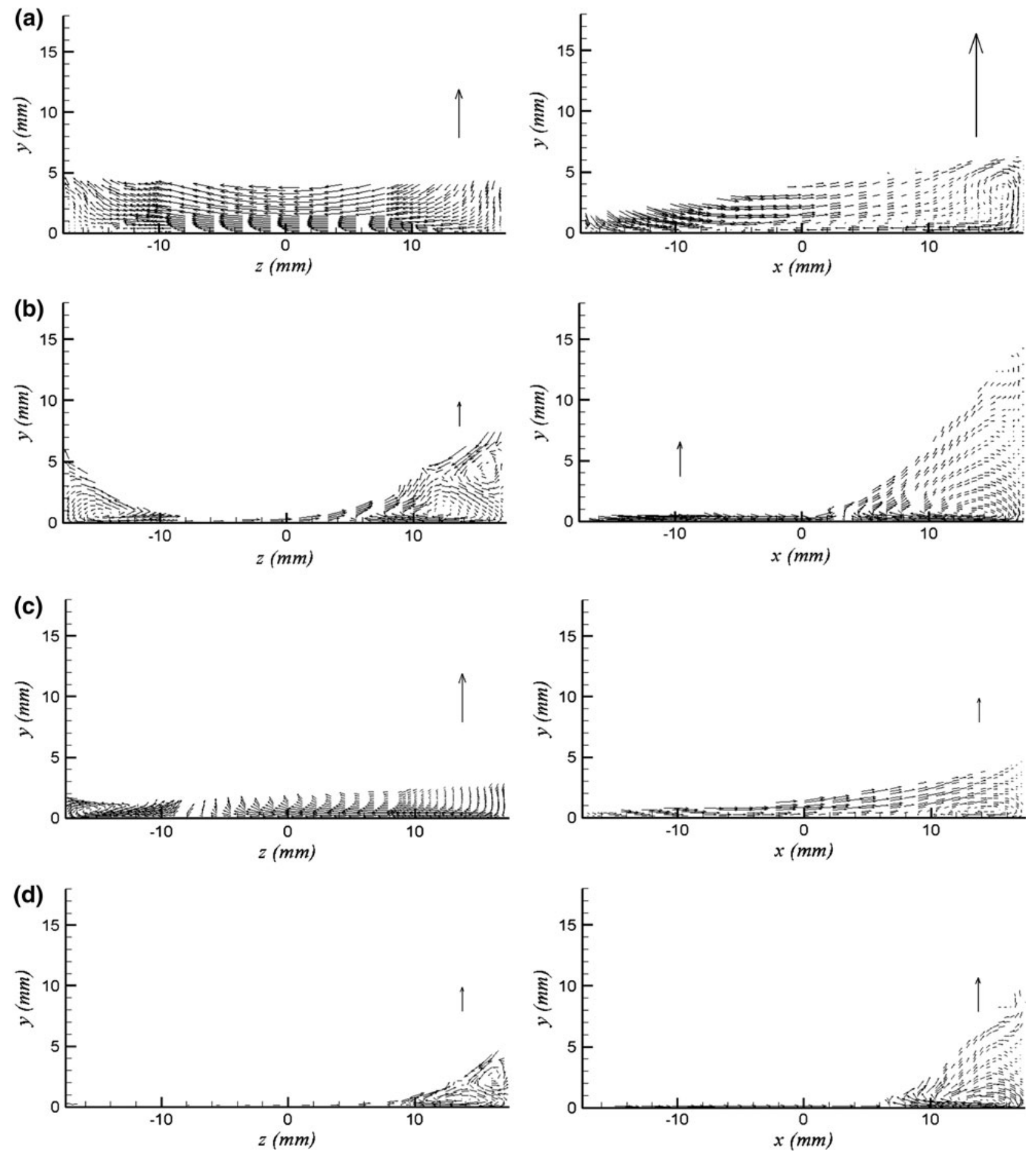

FIGURE 12. Vector plots of the in-plane velocity components in the liquid phase (a) $100 \mathrm{rpm}, 4 \mathrm{~mL}$; (b) $200 \mathrm{rpm}, 4 \mathrm{~mL}$; (c) $100 \mathrm{rpm}, 2 \mathrm{~mL}$; and (d) $200 \mathrm{rpm}, 4 \mathrm{~mL}$. The reference vector magnitude is $0.1 \mathrm{~m} / \mathrm{s}$.

weaker and Stokes' approximation yields satisfactory results.

The maximum level and the standard deviation of the fluctuations of the shear level magnitude are shown in Figs. $8 \mathrm{~b}$ and $8 \mathrm{c}$, respectively. Figure $8 \mathrm{c}$ shows that at each radius from the center of the well, how diverse the mean wall shear values are over a cycle. As indicated above, the maximum shear is associated with the passage of the bifurcation lines in the surface stress field. Near the distal walls, the induced secondary motion gives rise to strong variations in the shear levels. The large peaks observed near the distal corners in Fig. 8b for $\Omega=200 \mathrm{rpm}$ correlate well with the location of the near-wall vortex cores observed in Fig. 12b. At 
(a)

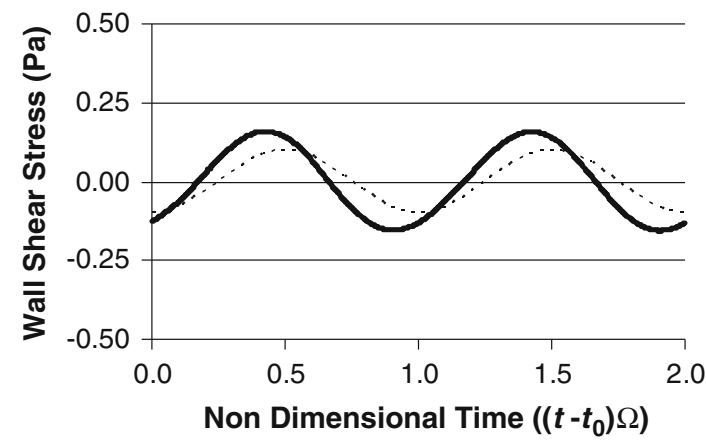

(b)

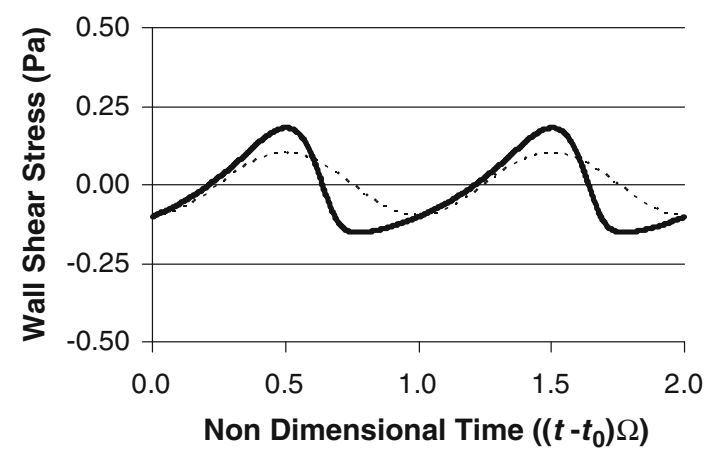

(c)

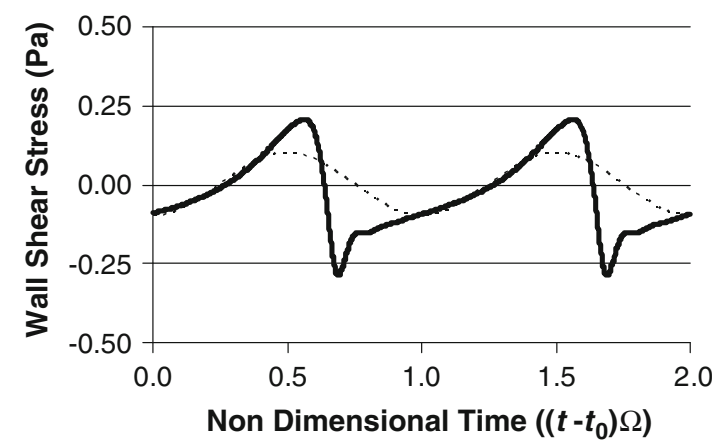

FIGURE 13. Time series of $z$-component of wall shear stress for $100 \mathrm{rpm}, 4 \mathrm{~mL}$ at $z=0$ (a) $x=1 \mathrm{~mm}$; (b) $x=6 \mathrm{~mm}$; (c) $x=12 \mathrm{~mm}$. At this locations the $z$-component of wall shear stress is equal to the magnitude of tangential wall shear stress but of opposite sign. The z-component of the velocity of the well is shown by dashed line.

$\Omega=100 \mathrm{rpm}, 2 \mathrm{~mL}$ (Fig. 12c), the vortex is weaker and correspondingly the peak shear stress values. Recall that the bifurcation line is located at the leading edge where the elevated fluid region (containing the semi-torus vortex) meets the thin fluid layer in the trough (Fig. 10). The deepest extent (smallest $r$ ) of the bifurcation line, indicated by arrows in Figs. 8a and 8c corresponds to a local minimum in $\tau_{\mathrm{w}}$ and a local maximum in the fluctuation levels (Standard deviation, SD).

\section{Biological Case Studies}

In this section, it will be shown that earlier observations on the orientation of endothelial cells (Dardik et $a l^{7}$ ) or the distribution of bacterial biofilms (Kostenko et al. ${ }^{15}$ ) in experiments conducted in similar culture well plates can be directly related to the wall shear stress distribution and flow topology analysed in this study. These results help to understand the underlying mechanisms leading to the observed differences between various locations of the culture plate.

(1) Dardik et al. ${ }^{7}$ exposed endothelial cells (EC) to surface shear stresses inside a six-well plate (similar to that in this study) on an orbital shaker at $210 \mathrm{rpm}$ and $2 \mathrm{~mL}$ of medium. The orientation of the EC at the well center and periphery were compared. They observed that EC in the periphery of the culture well were aligned along an angle of $\beta=34^{\circ} \pm 6^{\circ}$ from the line tangential to the edge of the well, while the EC observed in the center of the culture well were randomly aligned (Fig. 14).

The distribution of the instantaneous inclination angle of the surface shear stress vectors, measured relative to a radius:

$$
\alpha=\tan ^{-1}\left(-\frac{\tau_{\theta}}{\tau_{\mathrm{r}}}\right) \equiv 90^{\circ}-\beta
$$

where the $\tau_{\theta}$ and $\tau_{\mathrm{r}}$ indicate the tangential and radial components of the surface shear stress, is shown as colored contours for an arbitrary time point in Fig. 15a. Since the entire flow field rotates about the center of the plate with a constant rate, $\Omega$, it is possible to construct a scatter plot of $\alpha$ (sampled at equal time intervals) for each radial location, $r$, as shown in Fig. 15b. In the center region, as defined by Dardik et al., ${ }^{7} \alpha$ is independent of $r$ and uniformly distributed. A histogram of $\alpha$ constructed for all points in the center region, shown in Fig. 16a, suggests that EC throughout the center region are exposed to $\alpha$ ranging from $-90^{\circ}$ to $90^{\circ}$ with nearly equal probability. Hence, it is expected that the EC will be randomly oriented through the center region as found by Dardik et al. ${ }^{7}$

In contrast, in the periphery region $(9 \mathrm{~mm}<r<$ $16 \mathrm{~mm})$, a preferential clustering in the range $45^{\circ}<\alpha<60^{\circ}\left(30^{\circ}<\beta<45^{\circ}\right)$ can be deduced from the scatter plot of Fig. 16b. The histogram of Fig. 16b shows that over the entire periphery region, the shear stress is locally oriented in the range $\alpha=55^{\circ} \pm 10^{\circ}$ with significantly greater likelihood. Dardik et al. ${ }^{7}$ estimated an EC orientation angle of $\alpha=56^{\circ} \pm 6^{\circ}$ in the periphery region. These combined results indicate a strong correlation between the EC orientation and the most likely orientation of the local surface shear stress vector.

(2) In our earlier study (Kostenko et al. ${ }^{15}$ ), it was observed that the methicillin-resistant Staphylococcus aureus (MRSA) biofilms deposition varied radially across the well and more deposition occurred in the 


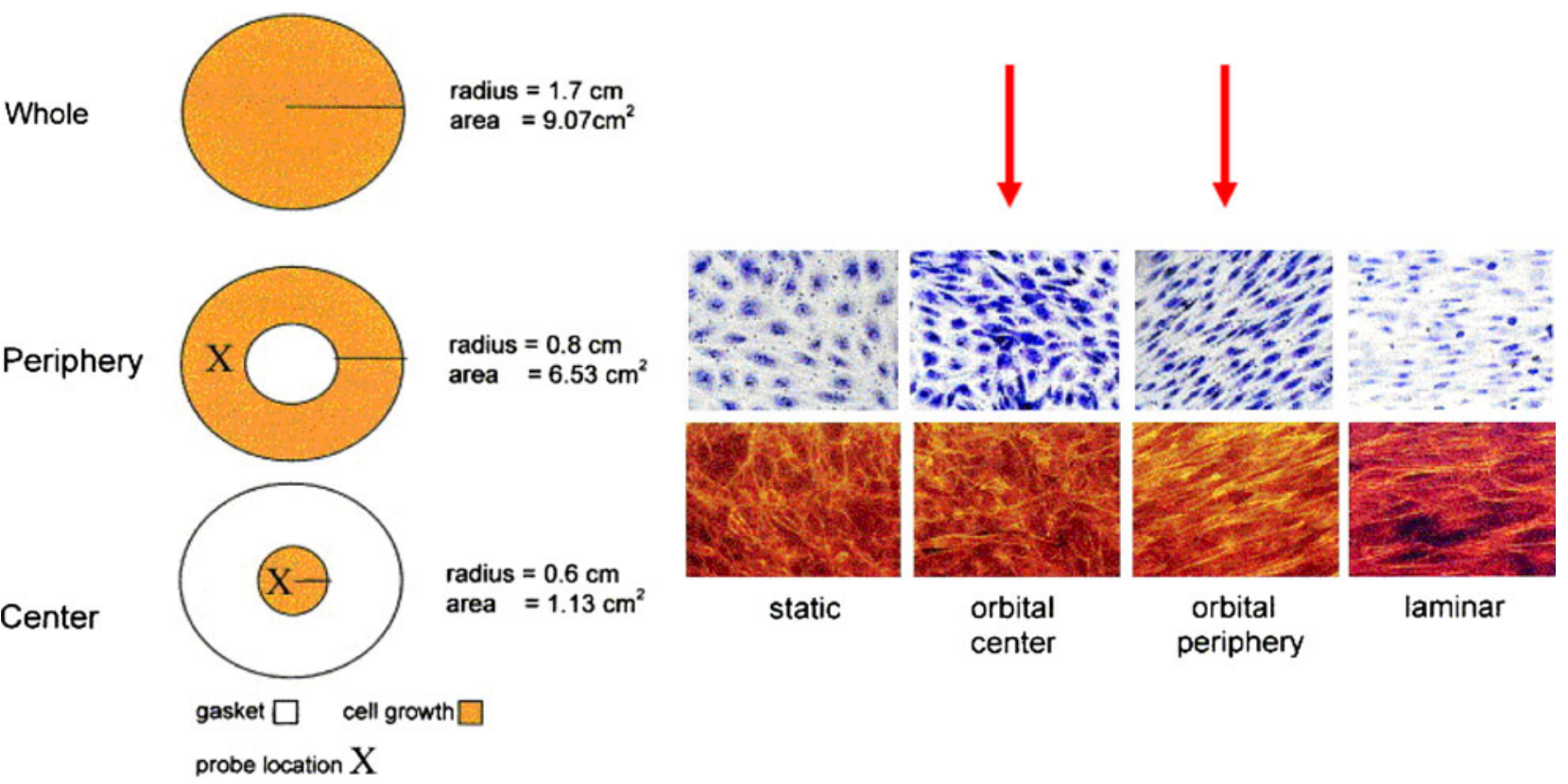

FIGURE 14. (a) Diagram of differential cell seeding (b) Morphology of ECs after 5 days of culture, ECs exposed to orbital motion at the center and periphery of culture well are marked by arrow. Figure from Dardik et al. ${ }^{7}$ with some changes.

periphery area compared to the center regions. Figure 17 a shows direct density measurements in terms of Colony Forming Units (CFU) at three radial locations. Figure $17 \mathrm{~b}$ shows the radial distribution of optical density (OD). The OD is directly proportional to the biofilm density. (Additional details on methodology are provided in Kostenko et al. ${ }^{15}$ ) The observed deposition distribution is difficult to reconcile by considering only the wall shear stress distribution. For example, as shown in Fig. 8a, at all three radial locations $(r=1,6,12 \mathrm{~mm})$ reported in Fig. 17, although the mean and peak wall shear stress magnitudes are comparable for both 2 and $4 \mathrm{~mL}$ cases at $100 \mathrm{rpm}$, the biofilm deposition differs significantly between locations. In contrast, for both cases at $200 \mathrm{rpm}$, while the shear stress magnitudes at $r=12$ are significantly higher than at $r=6 \mathrm{~mm}$, the biofilm deposition is similar.

Trying to relate the amount of biofilm accumulation to the shear stress levels leads to inconsistencies when comparing results from different studies as is discussed in Salek et al. ${ }^{25}$ It is, however, possible to relate these bacterial biofilm deposition patterns to the flow topology. The deposition is greatest in the region of the semi-torus vortex, which is swept by the negative (converging) bifurcation line making the leading front of the vortex (see Figs. 9, 10). In Fig. 17b, the deepest extent of the bifurcation line is indicated by an arrow. Thus, regions of larger radius are swept by the bifurcation line and are characterized by higher OD (i.e., biofilm density) than in the central regions. In the central regions of the plate (not swept by the bifurcation line), the fluid layer is thin and the flow is consistently directed toward the bifurcation line. The suspended bacteria in the central region are transported to the semi-torus vortex. The flow inside the vortex is characterized by a strong recirculation (e.g., Fig. 12), effectively trapping bacteria entering this region and thereby increasing the concentration of suspended bacteria. The microorganisms can be transported to the substratum through transport by the flow (Abelson and Denny $^{1}$ ) and settle. The entrainment of the free-surface layers into the recirculation regions can effectively maintain the oxygen supply, allowing bacteria to grow in the nutrient-rich medium.

Changes in biofilm morphology are often of interest as these correlate with susceptibility. ${ }^{15}$ Whereas differences in biofilm accumulation can be expanded in terms of the flow topology, the shear stress distribution also plays an important role in determining biofilm morphology. Figure 18 shows the typical morphotypes observed, and their location on the plates are summarized in Table 1.

The biofilms forming in the central region differ from those formed in the semi-torus region (i.e., region swept by the bifurcation line). At the lower rotational rate $(\Omega=100 \mathrm{rpm})$, the biofilms form low density, randomly distributed clumps in the central regions, while in the region swept by the bifurcation lines, biofilms form higher density, monolayer webs. For $\Omega=200 \mathrm{rpm}$, multilayered webs are observed in the central and distal regions. In the semi-torus region, the 
biofilm is compact and structure more complex (cup). Upon comparison of Table 1 and Fig. 8, these changes in morphology are not simply related to the density or
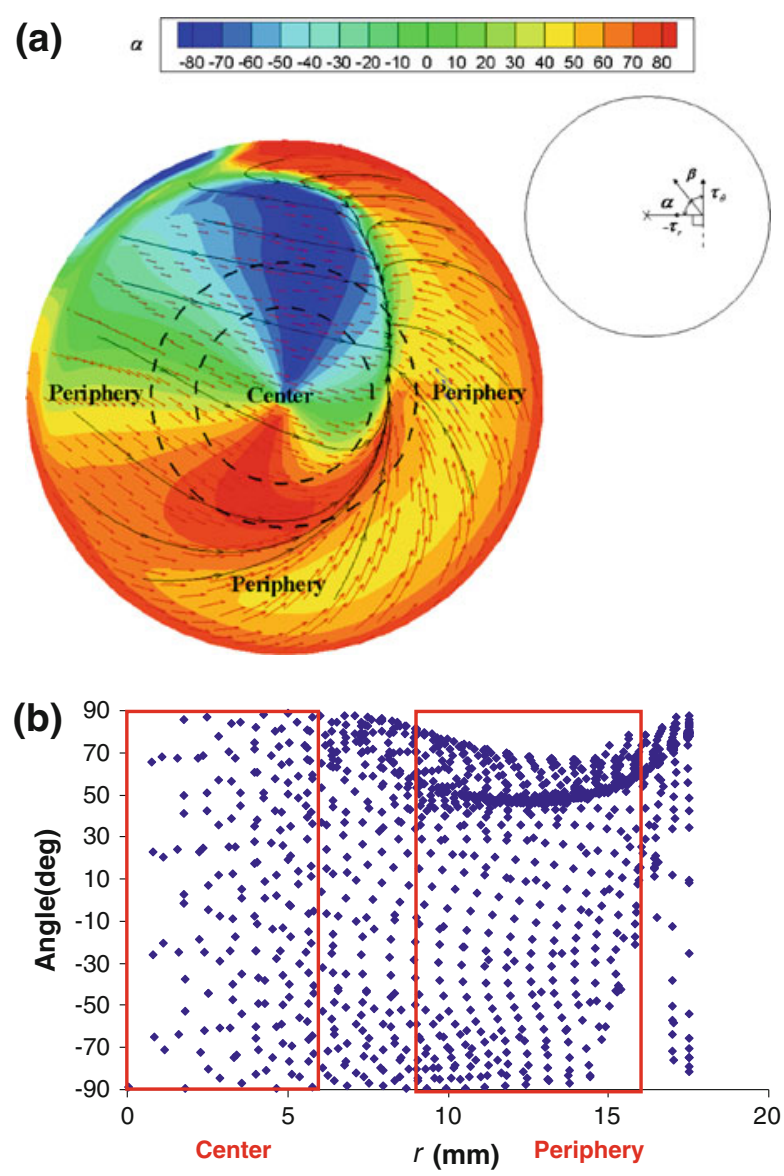

FIGURE 15. (a) Instantaneous iso-contours of the angle of the surface shear stress vectors, $\alpha$, with vectors superimposed. Regions investigated by Dardik et al. ${ }^{6}$ indicated by arrows. $\beta$ as defined per Dardik; $\alpha=90^{\circ}-\beta$ in this study; (b) scatter plot of shear stress angle $\alpha$ sampled during a cycle. Regions investigated by Dardik et al. ${ }^{7}$ (center and periphery) are indicated.

\section{(a)}

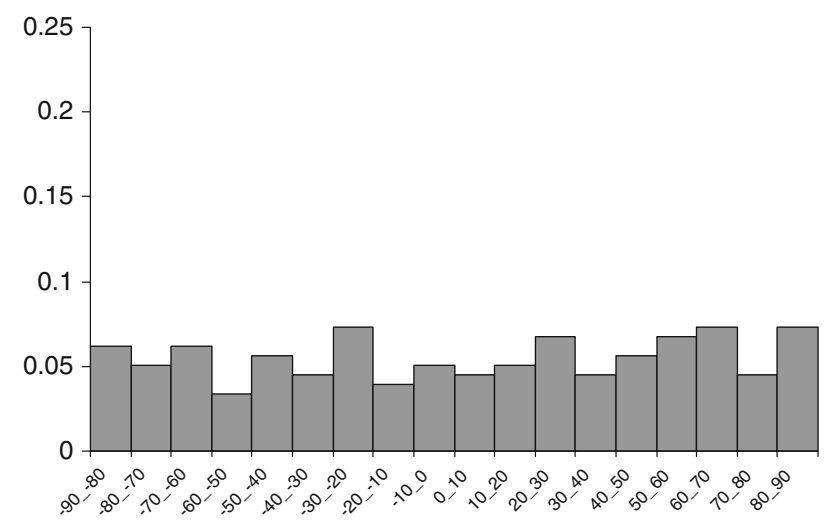

accumulation, but appear related to the fluctuation levels (or maximum value) of the shear stress magnitude. For example, the web morphology is observed in the semi-torus region at $100 \mathrm{rpm}$ and in the central region at $200 \mathrm{rpm}$, for which the fluctuation and maximum shear stress levels are comparable. Note the
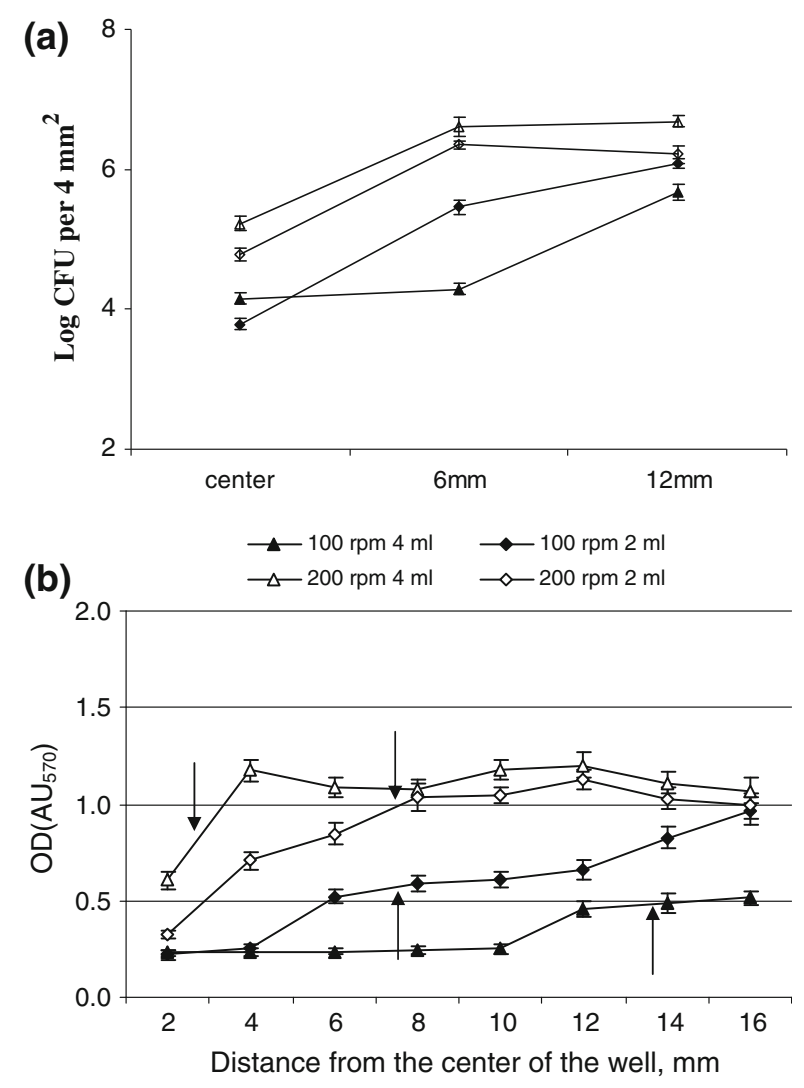

FIGURE 17. (a) Number MRSA cells accumulated within biofilms developed around either the center or 6 and $12 \mathrm{~mm}$ away from the center under different rotational speeds and volume of fluids (Kostenko et al. ${ }^{15}$ ); (b) the radial distribution of optical density (OD).

(b)

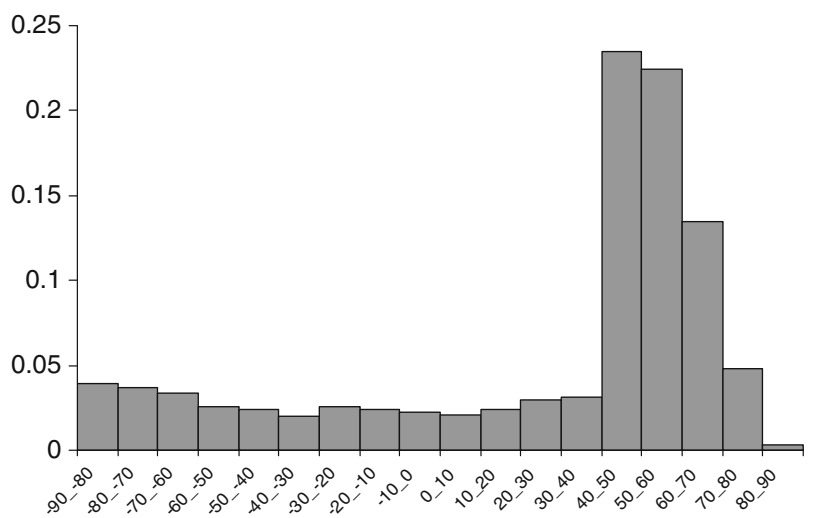

FIGURE 16. Histogram of the surface shear stress angle $\alpha$ calculated in this study for the regions in the (a) center and (b) periphery of Dardik et al. ${ }^{7}$. 

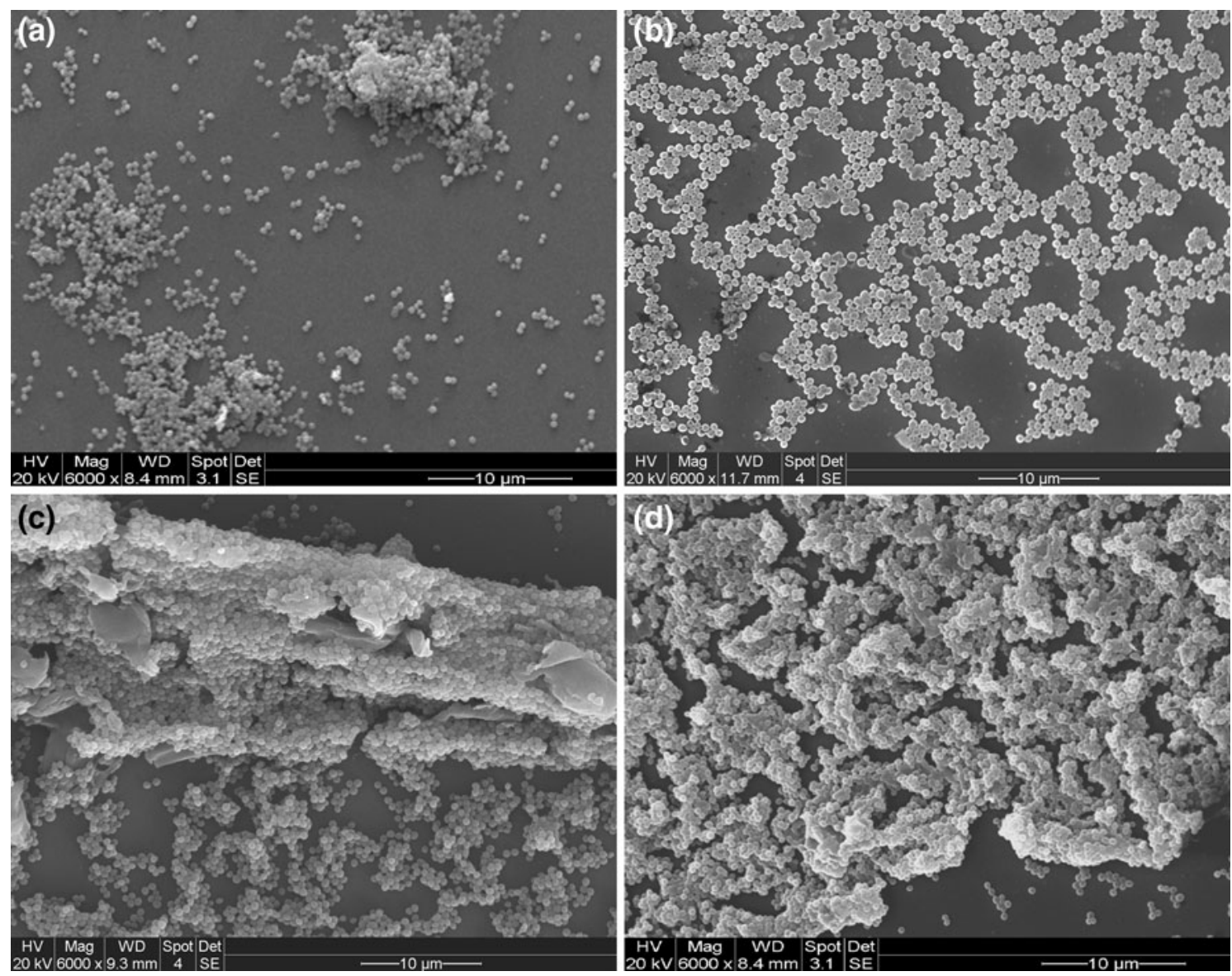

FIGURE 18. Representative samples of MRSA morphotypes found for (a) $100 \mathrm{rpm}$ in central region; (b) $100 \mathrm{rpm}$ in peripheral region; (c) $200 \mathrm{rpm}$ in peripheral region; (d) $200 \mathrm{rpm}$ in central region.

TABLE 1. Summary of the typical biofilm morphotypes and their location on the plates.

\begin{tabular}{lllllllll}
\hline & \multicolumn{7}{c}{ Radial distance from the center of the plate } \\
\cline { 2 - 8 } Experimental conditions & $2 \mathrm{~mm}$ & $4 \mathrm{~mm}$ & $6 \mathrm{~mm}$ & $8 \mathrm{~mm}$ & $10 \mathrm{~mm}$ & $12 \mathrm{~mm}$ & $14 \mathrm{~mm}$ & $16 \mathrm{~mm}$ \\
\hline $100 \mathrm{rpm} 4 \mathrm{~mL}$ & Clumps & Clumps & Clumps & Clumps & Clumps & Web & Web & Web \\
$100 \mathrm{rpm} 2 \mathrm{~mL}$ & Clumps & Clumps & Web & Web & Web & Web & Web & Web \\
$200 \mathrm{rpm} 4 \mathrm{~mL}$ & Multilayer web & Cup & Cup & Cup & Cup & Cup & Cup & Multilayer web \\
$200 \mathrm{rpm} 2 \mathrm{~mL}$ & Multilayer web & Cup & Cup & Cup & Cup & Cup & Multilayer web & Multilayer web \\
\hline
\end{tabular}

work is being pursued with the aim to distinguish between the influence of fluctuation and maximum shear stress levels.

\section{CONCLUSION}

The present study has shown that the fluid motion and wall shear stress distribution within a cylindrical well undergoing orbital translation in a horizontal plane is controlled by the liquid medium volume, $V$, the orbital radius of gyration, $R_{\mathrm{g}}$, and angular speed, $\Omega$. For operating conditions typical of microbiological experiments using orbital shakers, the fluid undergoes a solid body rotation, at the rotational speed $\Omega$, about the center of the well. Consequently, for a fixed point on the well surface, the wall shear stress, $\tau_{\mathrm{w}}$, fluctuates periodically at the frequency $\Omega$ and the mean and standard deviation of $\tau_{\mathrm{w}}$ are axisymmetric. The periodic fluctuations are generally not sinusoidal due to the presence of secondary flows, which arise in regions of higher liquid (free surface) elevation and are responsible for the observed radial distribution of the mean wall shear stress as well as the standard deviation. The local liquid elevation, $h$, appears to be governed by inertial effects and can be approximated based on a 
simplified analytical model as a function of $V, \Omega$, and $R_{\mathrm{g}}$.

This study is beneficial for interpreting results from biological experiments where the mean or fluctuating shear stress levels are important and also allows for systematic comparisons between different experiments. As examples, the orientation of endothelial cells and deposition patterns of bacterial biofilms have been related to the flow topology and wall shear stress behavior. The results suggest that the shear stress is not a sufficient factor to interpret the observations occurring in different experiments, but when it is linked to the topology of flow field, it helps to understand the underlying mechanisms of different biological processes.

\section{ACKNOWLEDGMENTS}

The authors recognize valuable discussions with Dr. Victoria Kostenko. M. Mehdi Salek would like to acknowledge Alberta Ingenuity Fund (AIF), now part of Alberta Innovates Technology Futures for their support through a scholarship. This research has been enabled by the use of computing resources provided by WestGrid and Compute/Calcul Canada.

\section{REFERENCES}

\footnotetext{
${ }^{1}$ Abelson, A., and M. Denny. Settlement of marine organisms in flow. Annu. Rev. Ecol. Syst. 28:317-339, 1997.

${ }^{2}$ Azevedo, N. F., A. R. Pinto, N. Reis, C. W. Keevil, and M. J. Vieira. Shear stress, temperature and inoculation concentration influence on the adhesion of water-stressed Helicobacter pylori to stainless steel 304. Appl. Environ. Microbiol. 72:2936-2941, 2006.

${ }^{3}$ Berson, R. E., M. R. Purcell, and M. K. Sharp. Computationally determined shear on cells grown in orbiting culture dishes. Adv. Exp. Med. Biol. 614:189-198, 2008.

${ }^{4}$ Bryers, J. D. Medical biofilms. Biotechnol. Bioeng. 100:1-18, 2008.

${ }^{5}$ Byrd, J. J., and T. M. Powledge. The Complete Idiots Guide to Microbiology. New York: Alpha, pp. 197-201, 2006.

${ }^{6}$ Chen, M. J., Z. Zhang, and T. R. Bott. Effects of operating conditions on the adhesive strength of Pseudomonas fluorescens biofilms in tubes. Colloids Surf. B 43:61-71, 2005.

${ }^{7}$ Dardik, A., L. Chen, J. Frattini, H. Asada, F. Haziz, F. Kudo, and B. Sumpio. Differential effects of orbital and laminar shear stress on endothelial cells. J. Vasc. Surg. 41(5):869-880, 2005.

${ }^{8}$ Donlan, R., and J. Costerton. Biofilms: survival mechanisms of clinically relevant microorganisms. Clin. Microbiol. Rev. 15:167-193, 2002.

${ }^{9}$ Fluent Manual, Fluent 6.3 FLUENT Inc., 2006.

${ }^{10}$ Greenspan, H. P., and L. N. Howard. On a time-dependent motion of a rotating fluid. J. Fluid Mech. 17:385-404, 1963.

${ }^{11}$ Greenspan, H. P. On the general theory of contained rotating fluid motion. J. Fluid Mech. 22(3):449-462, 1965.
}

${ }^{12}$ Haga, M., A. Yamashita, J. Paszkowiak, B. E. Sumpio, and A. Dardik. Oscillatory shear stress increases smooth muscle cell proliferation and Akt phosphorylation. J. Vasc. Surg. 37(6):1277-1284, 2003.

${ }^{13}$ Hopfinger, E. J., F. K. Browand, and Y. Gagne. Turbulence and waves in rotating tanks. J. Fluid Mech. 125:505534, 1982.

${ }^{14}$ Hopfinger, E. J., and G. J. F. van Heijst. Vortices in rotating fluids. Ann. Rev. Fluid Mech. 25:241-289, 1993.

${ }^{15}$ Kostenko, V., M. M. Salek, P. Sattari, and R. J. Martinuzzi. Staphylococcus aureus biofilm formation and tolerance to antibiotics in response to oscillatory shear stresses of physiological levels. FEMS Immunol. Med. Microbiol. 59:421-431, 2010.

${ }^{16}$ Kraiss, L. W., A. S. Weyrich, N. M. Alto, D. A. Dixon, T. M. Ennis, V. Modur, T. M. McIntyre, S. M. Prescott, and G. S. Zimmerman. Fluid flow activates a regulator of translation, p70/p85 S6 kinase, in human endothelial cells. Am. J. Physiol. Heart Circ. Physiol. 278:H1537-H1544, 2000.

${ }^{17}$ Ley, K., E. Lundgren, E. M. Berger, and K.-E. Arfors. Shear-dependent inhibition of granulocyte adhesion to cultured endothelium by dextran sulfate. Blood 73:13241330, 1989.

${ }^{18}$ MacLeod, S. M., and D. J. Stickler. Species interactions in mixed-community crystalline biofilms on urinary catheters. Med. Microbiol. 56(Pt 11):1549-1557, 2007.

${ }^{19}$ Meda, M. S., A. J. Lopez, and A. Guyot. Candida inferior vena cava filter infection and septic thrombophlebitis. Br. J. Radiol. 80(950):e48-e49, 2007.

${ }^{20}$ Murray, T. S., M. Egan, and B. I. Kazmierczak. Pseudomonas aeruginosa chronic colonization in cystic fibrosis patients. Curr. Opin. Pediatr. 19(1):83-88, 2007.

${ }^{21}$ Percival, S. L., and P. G. Bowler. Biofilms and their potential role in wound healing. Wounds 16:234-240, 2004.

${ }^{22}$ Phillips, P. L., E. Sampson, Q. Yang, P. Antonelli, A. Progulske-Fox, and G. Schultz. Bacterial biofilms in wounds. Wound Healing South Afr. 1:10-12, 2008.

${ }^{23}$ Presterl, E., A. Lassnigg, M. Eder, S. Reichmann, A. M. Hirschl, and W. Graninger. Effects of tigecycline, linezolid and vancomycin on biofilms of viridans streptococci isolates from patients with endocarditis. Int. J. Artif. Organs 30(9):798-804, 2007.

${ }^{24}$ Purevdorj, B., J. W. Costerton, and P. Stoodley. Influence of hydrodynamics and cell signaling on the structure and behavior of Pseudomonas aeruginosa biofilms. Appl. Environ. Microbiol. 68:4457-4464, 2002.

${ }^{25}$ Salek, M. M., S. Jones, and R. J. Martinuzzi. The influence of flow cell geometry related shear stresses on the distribution, structure and susceptibility of Pseudomonas aeruginosa 01 biofilms. Biofouling 25:711-725, 2009.

${ }^{26}$ Sattari, P. Design and Fabrication of an Optical Nonintrusive Shear Rate Sensor. M.Sc. Thesis, Department of Mechanical and Manufacturing Engineering, University of Calgary, 2008.

${ }^{27}$ Sherman, F. S. Viscous Flow. New York: McGraw-Hill, pp. 140-141, 1990.

${ }^{28}$ Sillankorva, S., P. Neubauer, and J. Azeredo. Pseudomonas fluorescens biofilms subjected to phage phiIBB-PF7A. BMC Biotechnol. 8:79, 2008.

${ }^{29}$ Simões, M., M. O. Pereira, S. Sillankorva, J. Azeredo, and M. J. Vieira. The effect of hydrodynamic conditions on the phenotype of Pseudomonas fluorescens biofilms. Biofouling 23:249-258, 2007. 
${ }^{30}$ Simões, M., M. O. Pereira, and M. J. Vieira. The role of hydrodynamic stress on the phenotypic characteristics of single and binary biofilms of Pseudomonas fluorescens. Water Sci. Technol. 55:437-445, 2007.

${ }^{31}$ Sousa, C., M. Henriques, J. Azeredo, P. Teixeira, and R. Oliveira. Staphylococcus epidermidis glucose uptake in biofilm versus planktonic cells. World J. Microbiol. Biotechnol. 24:423-426, 2008.

${ }^{32}$ Stoodley, P., I. Dodds, J. D. Boyle, and H. M. Lappin-Scott. Influence of hydrodynamics and nutrients on biofilm structure. J. Appl. Microbiol. 85:S19-S28, 1999.
${ }^{33}$ White, F. M. Fluid Mechanics (6th ed.). New York: McGraw-Hill, pp. 164-166, 2008.

${ }^{34}$ Wilson, D. W., J. A. Scalf, S. Forouhar, R. Muller, F. Taugwalder, M. Gharib, D. Fourguette, and D. Modarress. Diffractive optic fluid shear stress sensor. In: Trends in Optics and Photonics, Diffractive Optics and Micro-Optics, Vol. 41, edited by T. Li. Washington, DC: Optical Society of America, 2000, pp. 306-308.

${ }^{35}$ Youngs, D. L. Time-dependent multi-material flow with large fluid distortion. In: Numerical Methods for Fluid Dynamics, edited by K. W. Morton, and M. J. Baines. New York: Academic, 1982. 